Journal of

Synchrotron

Radiation

ISSN 0909-0495

Received 15 January 2013

Accepted 3 July 2013

\section{The Materials Science beamline upgrade at the Swiss Light Source}

P. R. Willmott, ${ }^{\mathrm{a} *}$ D. Meister, ${ }^{\text {a }}$ S. J. Leake, ${ }^{\mathrm{a}}$ M. Lange, ${ }^{\mathrm{a}}$ A. Bergamaschi, ${ }^{\mathrm{a}}$ M. Böge, ${ }^{\mathrm{a}}$ M. Calvi, ${ }^{a}$ C. Cancellieri, ${ }^{a}$ N. Casati, ${ }^{a}$ A. Cervellino, ${ }^{a}$ Q. Chen, ${ }^{a}$ C. David, ${ }^{b}$ U. Flechsig, ${ }^{a}$ F. Gozzo, ${ }^{c}$ B. Henrich, ${ }^{a}$ S. Jäggi-Spielmann, ${ }^{a}$ B. Jakob, ${ }^{a}$ I. Kalichava, ${ }^{a}$ P. Karvinen, ${ }^{b}$ J. Krempasky, ${ }^{a}$ A. Lüdeke, ${ }^{a}$ R. Lüscher, ${ }^{a}$ S. Maag, ${ }^{d}$ C. Quitmann, ${ }^{a} \neq$ M. L. Reinle-Schmitt, ${ }^{a}$ T. Schmidt, ${ }^{a}$ B. Schmitt, ${ }^{a}$ A. Streun, ${ }^{a}$ I. Vartiainen, M. Vitins, ${ }^{d}$ X. Wang ${ }^{a}$ and R. Wullschleger ${ }^{a}$

${ }^{\mathbf{a}}$ Swiss Light Source, Paul Scherrer Institut, CH-5232 Villigen, Switzerland, ${ }^{\mathbf{b}}$ Laboratory for Microand Nanotechnology, Paul Scherrer Institut, CH-5232 Villigen, Switzerland, ${ }^{\mathbf{c}}$ Excelsus Structural Solutions SPRL, B-1150 Brussels, Belgium, and d Department of Machine Engineering, Paul Scherrer Institut, CH-5232 Villigen, Switzerland. E-mail: philip.willmott@psi.ch

The Materials Science beamline at the Swiss Light Source has been operational since 2001. In late 2010, the original wiggler source was replaced with a novel insertion device, which allows unprecedented access to high photon energies from an undulator installed in a medium-energy storage ring. In order to best exploit the increased brilliance of this new source, the entire front-end and optics had to be redesigned. In this work, the upgrade of the beamline is described in detail. The tone is didactic, from which it is hoped the reader can adapt the concepts and ideas to his or her needs.

Keywords: beamline upgrade; undulator radiation; X-ray optics; powder diffraction; surface diffraction; coherent $X$-ray diffraction imaging.

\section{Introduction}

The Materials Science (MS) beamline at the Swiss Light Source (SLS) was originally conceived to provide hard X-rays in the energy range 5-40 keV (Patterson et al., 2005) and serve experiments in powder diffraction (PD), surface diffraction (SD) and computed tomography. As such, it was the only insertion-device beamline at the SLS to exceed a photon energy of $20 \mathrm{keV}$.

In 2000, it was only possible to access such high photon energies at the intermediate storage-ring electron energies of $\mathcal{E}=2.4 \mathrm{GeV}$ of the SLS by employing a so-called minigap wiggler (called 'W61' to indicate the magnets' period of $60.5 \mathrm{~mm}$ ), with a critical energy of $7 \mathrm{keV}$ and a deviation parameter $K=8.6$ at a gap size of $8 \mathrm{~mm}$ (Patterson et al., 2005). The transmitted flux at $12 \mathrm{keV}$ from the wiggler of

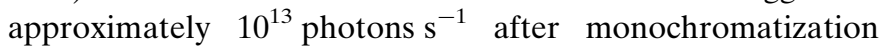
through a $\mathrm{Si}(111)$ double-crystal monochromator (DCM) was at best $30 \%$ efficient. This was due primarily to the large heat load on the first DCM crystal, which caused a thermal bump and consequent reduction in flux, despite the use of the dynamical correction system 'TORII' (Schulte-Schrepping et al., 1995). Another consequence of the high output power of W61 was the need to use a so-called rotating carbon filter to

† Present address: MAX IV Laboratory, Ole Römers väg 1, SE-223 63 Lund, Sweden. remove the strongly absorbed soft X-ray part of the wiggler spectrum below approximately $5 \mathrm{keV}$, amounting to $1.6 \mathrm{~kW}$, a power too high to be able to use a cooled diamond filter. This front-end component, as well as two beryllium windows, increased the effective source size to approximately $450 \mu \mathrm{m}$ (h) $\times 250 \mu \mathrm{m}(\mathrm{v})$.

Since 2000, however, undulator technology has matured, in particular with regards to magnet materials and in-vacuum technology. As described in detail in the next section, high photon energies can begin to be accessed at medium-energy storage rings, once radiation-hard small-period magnets with high maximum magnetic field strength $B_{0}$ become available. Such magnets were developed around 2003, and their implementation in undulator technology has allowed a considerable reduction of undulator-magnet period lengths from $19 \mathrm{~mm}$ to $14 \mathrm{~mm}$.

With the advent of this technological advance, it was decided in 2007 to replace W61 with an in-vacuum cryogenically cooled permanent-magnet undulator [CPMU (Hara et al., 2004)], having a magnet period $\lambda_{\mathrm{u}}=14 \mathrm{~mm}$, resulting in the CPMU being called 'U14'.

Because the beam divergence of U14, and hence the beam cross section at the optics hutch, is so much smaller than that of W61, new optics had to be designed (see §6). At the same time, the new design also took into account the possibility of introducing new techniques at the beamline, such as 
Table 1

SLS specifications.

\begin{tabular}{ll}
\hline Parameter & Value \\
\hline Storage-ring energy & $2.4 \mathrm{GeV}$ \\
Lorentz function & 4697 \\
Circumference & $288 \mathrm{~m}$ \\
Electron current & $400 \mathrm{~mA}$ (top-up operation) \\
Horizontal emittance & $5.5 \mathrm{~nm} \mathrm{rad}$ \\
Horizontal beta function & $1.38 \mathrm{~m} \mathrm{rad}^{-1}$ \\
Vertical emittance & $5 \mathrm{pm} \mathrm{rad}$ \\
Vertical beta function & $1.0 \mathrm{~m} \mathrm{rad}^{-1}$ \\
Electron-beam size (FWHM) & $204 \mu \mathrm{m}(\mathrm{h}) \times 5.3 \mu \mathrm{m}(\mathrm{v})$ \\
\hline
\end{tabular}

Table 2

Materials Science beamline specifications.

\begin{tabular}{ll}
\hline Parameter & Value \\
\hline Photon energy range & $5-40 \mathrm{keV}$ \\
Flux at $12 \mathrm{keV}$ & $>10^{13} \mathrm{photons} \mathrm{s}^{-1}$ \\
Energy resolution $\Delta E / E$ & $1.4 \times 10^{-4}$ \\
Focus at SD station $(1: 1)$ & Better than $200 \mu \mathrm{m}(\mathrm{h}) \times 30 \mu \mathrm{m}(\mathrm{v})$ \\
\hline
\end{tabular}

coherent X-ray diffraction imaging and micro Laue diffraction.

The paper is organized as follows: after a description of the specifications of the SLS storage ring, the U14 undulator and Materials Science beamline given in $\S 2$ and $\S 3$, details of the beamline layout, the front-end components and optics design are described in $\S 4, \S 5$ and $\S 6$, respectively. The performance of the beamline after the upgrade is covered in $\S 7$, and the impact of this on the two endstations, plus first results, are described in $\S 8$ and $\S 9$.

\section{Specifications for the SLS and the Materials Science beamline}

Since the last publication describing the MS beamline (Patterson et al., 2005), the storage-ring specifications have improved considerably in terms of vertical emittance and electron-beam size. This is due mostly to an exceedingly low coupling of the horizontal to the vertical emittance of $5 \times$ $10^{-4}$. Such coupling is caused primarily by slight misalignments of the focusing quadrupole magnets. The most important parameters are listed in Table 1.

The SLS runs in 'top-up' operation, in which the storage current is maintained to within $1 \%$ of the nominal value of $400 \mathrm{~mA}$. This feature is important in providing a constant $\mathrm{X}$-ray flux and stable beam position.

The specifications of the MS beamline are summarized in Table 2. The improved coherent fraction promised by undulator radiation should also be exploited. This feature sets stringent specifications on both the optics and on the vacuum quality; the gradual build-up of carbon deposits on the surface of the first crystal in the DCM, caused by the photo-induced cracking of carbon-containing gaseous species in the residual gas, can rapidly and adversely affect the quality of the wavefront. The residual gas pressure in the DCM was therefore specified as being less than $10^{-8}$ mbar.

\section{The U14 undulator}

\subsection{Theoretical design considerations}

The on-axis peak intensity $I_{n}$ of the $n$th harmonic of undulator radiation is given by

$$
I_{n}=\alpha N^{2} \gamma^{2} \frac{\Delta v}{v} \frac{I}{e} F_{n}(K)
$$

whereby $\alpha=1 / 137$ is the fine-structure constant, $\gamma$ is the Lorentz factor $\mathcal{E} / m c^{2}, \Delta v / v=1 / n N$ is the relative spectral bandwidth of the $n$th harmonic peak, $I$ is the current, $e$ is the elementary charge, $N$ is the number of magnet periods and $F_{n}(K)$ is the so-called tuning function, given by

$$
F_{n}(K)=\chi^{2} K^{2}\left[J_{(n-1) / 2}\left(K^{2} \chi / 4\right)-J_{(n+1) / 2}\left(K^{2} \chi / 4\right)\right]^{2},
$$

whereby

$$
\chi=n /\left(1+K^{2} / 2\right)
$$

and $J$ are Bessel functions of the first kind. $F_{n}(K)$ is plotted as a function of $K$ in Fig. 1(a). The deviation parameter $K$ is defined as

$$
K=\varphi_{\max } \gamma=\frac{e B_{0} \lambda_{\mathrm{u}}}{2 \pi m c}=0.934 \lambda_{\mathrm{u}}[\mathrm{cm}] B_{0}[\mathrm{~T}]
$$

whereby $\varphi_{\max }$ is the maximum angular excursion off-axis of the electrons passing through the undulator magnet array, $m$ is the electron rest mass, and $c$ is the speed of light in a vacuum (Willmott, 2011).
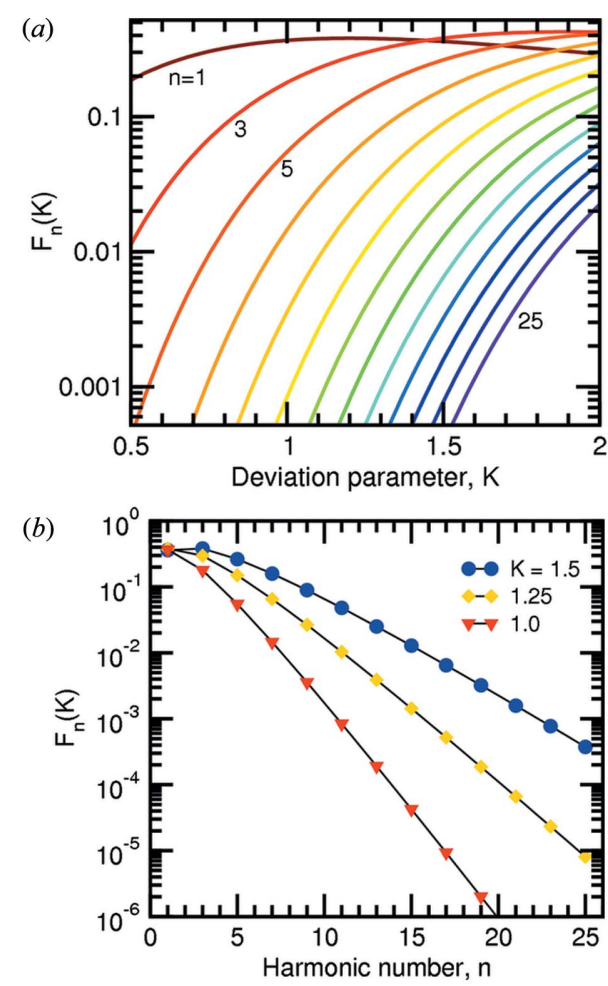

Figure 1

Plot of the tuning function $F_{n}(K)$ as a function of $(a) K$, for the odd harmonics between $n=1$ and 25; and $(b) n$, for three different values of $K$. 
One sees immediately from Fig. 1(a) that, even for relatively high values of $K \simeq 1.5, F_{n}(K)$, and hence also $I_{n}$, is three orders of magnitude smaller for $n \simeq 21$ than for the first few harmonics. Fig. $1(b)$ is a plot of $F_{n}(K)$ versus harmonic number $n$ for three values of $K$.

The condition for constructive interference on-axis in an undulator is given by

$$
n \lambda_{n}=\frac{\lambda_{\mathrm{u}}}{2 \gamma^{2}}\left(1+K^{2} / 2\right)
$$

or, in practical units,

$$
n \lambda_{n}[\AA]=\frac{13.056 \lambda_{\mathrm{u}}[\mathrm{cm}]}{\mathcal{E}^{2}[\mathrm{GeV}]}\left(1+K^{2} / 2\right) .
$$

Hence, for a given storage-ring energy $\mathcal{E}$, undulator magnet period $\lambda_{\mathrm{u}}$ and deviation parameter $K, n \lambda_{n}$ is a constant, which, according to Fig. 1(a), we would like to be as small as possible, in order to access those tuning curves with as low as possible value of $n$. On the other hand, we require $K$ to be as large as possible (Fig. 1b). The only possibility to achieve both these requirements is, therefore, to reduce $\lambda_{u}$ [equation (6)] and increase $B_{0}$ [equation (4)].

The previous shortest-period undulator at the SLS, U19, had $\lambda_{\mathrm{u}}=19 \mathrm{~mm}$. In order for the insertion device to remain an undulator, $K$ should not exceed 2.0 or thereabouts. Hence, from equation (4), the maximum magnetic field strength must be equal to or less than approximately 1.1 T. For the maximum $K$ of $2.0, n \lambda_{n}$ for U19 was equal to $12.92 \AA$ at the SLS. Accessing photon energies above $30 \mathrm{keV}$ thus required working on the 31st harmonic, for which the intensity is unacceptably low [see Fig. 1(a)]. Indeed, U19 at the protein crystallography beamline at the SLS operates only up to $19 \mathrm{keV}$ photon energy.

Because the U14 undulator is an in-vacuum insertion device, the gap size can in principle be closed to as little as $3.5 \mathrm{~mm}$ and the maximum magnetic field strength $B_{0}$ be as large as $1.5 \mathrm{~T}$ (Hara et al., 2004). This was measured as a function of gap size $g$ using a Hall probe (see Fig. 2). The resulting $K$ values [equation (4)] were then fit, yielding

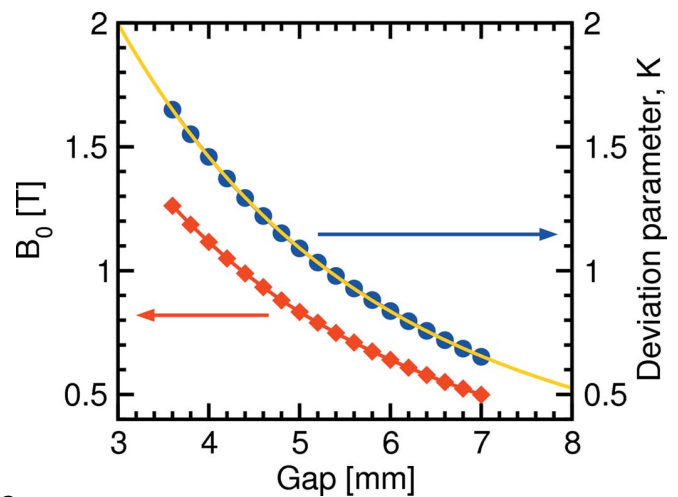

Figure 2

Plot of the maximum on-axis magnetic field $B_{0}$ and deviation parameter $K$ as a function of the U14 undulator gap size. The yellow solid curve is a best fit to $K$ [see equation (7)].
Table 3

U14 CPMU specifications compared with those of the W61 wiggler previously installed at the MS beamline and the U19 undulator installed

\begin{tabular}{|c|c|c|c|}
\hline Parameter & U14 & W61 & U19 at PX1 \\
\hline Overall length (m) & 1.680 & 1.906 & 1.82 \\
\hline Period length (mm) & 14 & 60.5 & 19 \\
\hline Number of poles $(2 N)$ & 240 & 63 & 192 \\
\hline Power output (W) & 3000 & 5700 & 2600 \\
\hline $\begin{array}{l}\text { Beam divergence } \\
{[\text { FWHM h }(\mu \mathrm{rad})} \\
\times \mathrm{v}(\mu \mathrm{rad})] \text { at } 12 \mathrm{keV}\end{array}$ & $144 \times 16.5$ & $2500 \times 230$ & $135 \times 25$ \\
\hline $\begin{array}{l}\text { Minimum magnetic gap } \\
(\mathrm{mm})\end{array}$ & 3.8 & 8 & 4.5 \\
\hline $\begin{array}{l}\text { Maximum deviation } \\
\text { parameter } K\end{array}$ & 1.46 & 8.6 & 2.46 \\
\hline $\begin{array}{l}\text { Beam size at DCM } \\
\text { [FWHM h }(\mathrm{mm}) \\
\times \mathrm{v}(\mathrm{mm})]\end{array}$ & $2.9 \times 0.33$ & $49 \times 4.5$ & $2.65 \times 0.49$ \\
\hline $\begin{array}{l}\text { Power on first DCM } \\
\text { crystal (W) }\end{array}$ & 157 & 733 & 137 \\
\hline Maximum field (T) & 1.26 & 1.84 & 1.39 \\
\hline Critical energy (keV) & 4.8 & 7.0 & 5.3 \\
\hline $\begin{array}{l}\text { Flux at } 12 \mathrm{keV} \\
\quad\left(\text { photons s}^{-1}\right)\end{array}$ & $2 \times 10^{13}$ & $10^{13}$ & $2 \times 10^{12}$ \\
\hline $\begin{array}{l}\text { Best focus }(1: 1) \\
{[\text { FWHM }(\mu \mathrm{m})]}\end{array}$ & $80 \times 30$ & $450 \times 300$ & - \\
\hline
\end{tabular}
at the PX1 beamline of the SLS.

$$
K=5.9 \exp \left(-5.55 \frac{g}{\lambda_{\mathrm{u}}}+2.31 \frac{g^{2}}{\lambda_{\mathrm{u}}^{2}}\right)
$$

In practice, the minimum gap size has been limited to $3.8 \mathrm{~mm}$.

From equation (4), the maximum value of $K$ is approximately 1.65 , and $n \lambda_{n}=7.49$. Accessing $30 \mathrm{keV}$ photons requires one to operate at the 17 th harmonic, for which the flux is an order of magnitude higher than that for U19 [see Fig. 1(a)].

\subsection{The U14 CPMU design}

A detailed description of the U14 CPMU design and performance is given elsewhere (Calvi et al., 2013). Here, the most important features are highlighted.

The undulator design parameters compared with those for the W61 wiggler and U19 undulator are summarized in Table 3. The magnet material chosen for U14 was Neomax S45SH, which has the alloy composition $\mathrm{Nd}_{2} \mathrm{Fe}_{14} \mathrm{~B}$. The remanent field of this material maximizes at approximately $140 \mathrm{~K}$ (Hara et al., 2004) and is higher than related commercial magnets. Importantly, demagnetization caused by exposure to high-energy electrons is almost completely suppressed at this temperature. While at room temperature the magnetic field strength drops by almost $30 \%$ after exposure to $1.5 \times 10^{15} 2 \mathrm{GeV}$ electrons, the same exposure at $140 \mathrm{~K}$ produces a drop in field strength of approximately $1 \%$. U14 is hence cryogenically cooled and maintained at $138 \pm 0.1 \mathrm{~K}$, by a combination of liquid- $\mathrm{N}_{2}$ cooling and heating elements. The temperature is measured at three positions (upstream, centre, downstream) on both the upper and lower undulator support structures. A second important aspect of this material is that, although the magnetic field strength only increases modestly when cooled to $140 \mathrm{~K}$, 
its coercivity increases by over a factor of two, allowing one to use it at small gap sizes.

The liquid- $\mathrm{N}_{2}$ pump system is also used to cool the first crystal of the DCM (see §6.2). This element, including the internal metallic bellows for the nitrogen flow, causes its thermally insulated goniometer stage to cool over approximately $100 \mathrm{~h}$ from room temperature to $290 \mathrm{~K}$. This has the sadly unavoidable consequence that experiments at high energy, for which the DCM crystals' Darwin widths are narrow, are relatively unstable during this cool-down period, as the goniometer slowly drifts thermally.

The short undulator period allows more magnet poles to be installed $(N=120$, compared with that of U19 at the SLS, for which $N=96$; see Table 3 ). From equation (1), we see that the brilliance of U14 is also enhanced by a factor of approximately 1.5 because of this aspect. Despite the complication of cooling the CPMU, it was possible to maintain a phase error of approximately $2.5^{\circ}$ between the useful gap sizes of 3.8-6 mm. Plots of the variation of the gap size with photon energy for the third to 19th harmonics, plus the theoretical flux, taking into account phase errors, are shown in Fig. 3, calculated using the $S R W$ code (Chubar et al., 1998; see also http://www.esrf.eu/ Accelerators/Groups/InsertionDevices/Software/SRW). Also included in the latter are experimentally determined fluxes, recorded at the SD station using the photon-counting Pilatus $100 \mathrm{k}$ detector, corrected for the various kapton, diamond, mylar and $\mathrm{Si}_{3} \mathrm{~N}_{4}$ components, the detector efficiency [Fig. 4(a)], the mirrors' capture cross-section (a factor which begins to

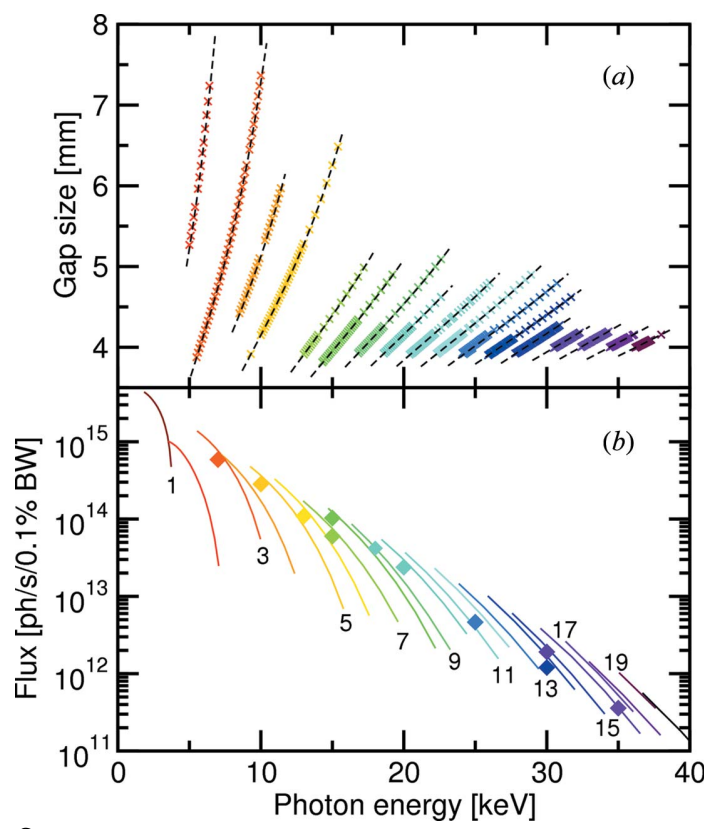

Figure 3

Plots of $(a)$ the experimental gap sizes (crosses) as a function of photon energy between the third and 19th harmonics, plus the theoretical curves, as calculated with $S R W$ (dashed lines); and (b) the theoretical photon flux after monochromatization by the DCM for all harmonics from the first to the 20th, also calculated using $S R W$. The diamond symbols are the experimentally determined fluxes, taking into account all correction factors associated with the beamline components and detector efficiency. Their colours indicate at which harmonic the flux was recorded.
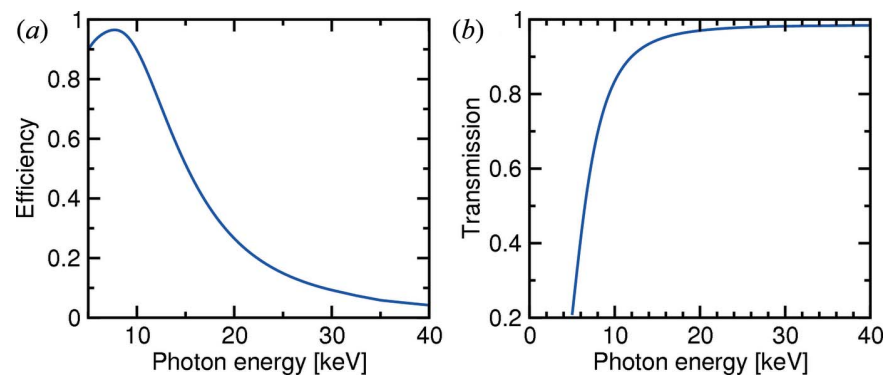

Figure 4

(a) Plot of the quantum efficiency of the Pilatus 100k pixel detector used at the SD station, including absorption due to the protective layer of aluminium, mylar and $\mathrm{Si}_{3} \mathrm{~N}_{4}$ in front of the detector, over the range 5$40 \mathrm{keV}$. (b) Plot of the transmission spectrum of X-rays through $236 \mu \mathrm{m}$ of diamond (density $=3.51 \mathrm{~g} \mathrm{~cm}^{-3}$ ) in the range $5-40 \mathrm{keV}$.

become important above approximately $20 \mathrm{keV}$ ) and the bandwidth of the Si(111) crystals in the DCM (see below). At lower energies, the experimentally obtained flux is $70 \%$ or better compared with theory, dropping to approximately $50 \%$ above $25 \mathrm{keV}$.

Of more direct interest to the user, however, is the actual number of photons per second that he/she can expect after the optics hutch, as a function of energy. This is shown in Fig. 5. For purposes of comparison, the total flux of the old wiggler insertion device W61 is also plotted. At the lowest photon energies, the flux from U14 is orders of magnitude higher, dropping to about $50-75 \%$ of the W61 flux at the highest photon energies. Given that the unfocused beam of U14 is over 200 times smaller than that of W61, the areal flux density of U14 is thus over two orders of magnitude higher.

At the SD station, the best focus is approximately $130 \mu \mathrm{m}$ (h) $\times 40 \mu \mathrm{m}(\mathrm{v})$, or about 20 times smaller than previously obtainable (see the inset of Fig. 5) recorded at $9 \mathrm{keV}$.

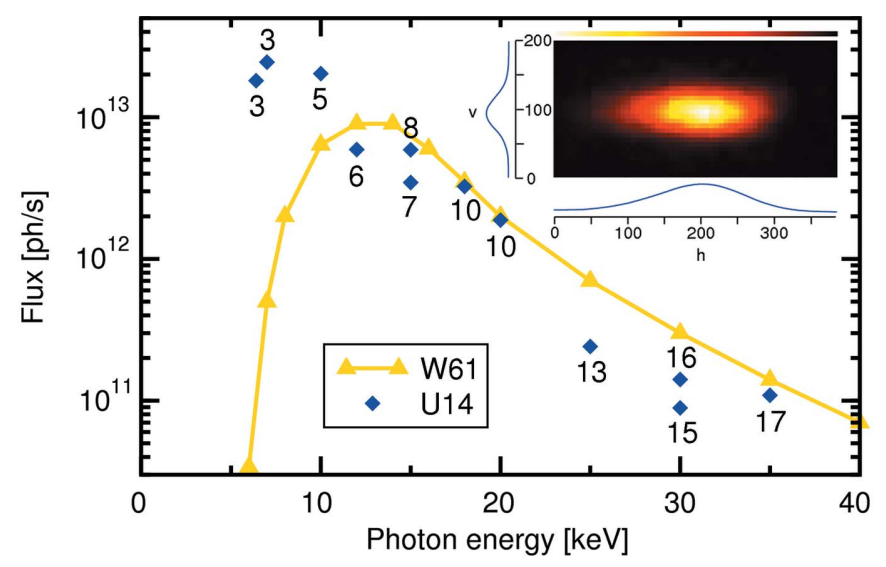

Figure 5

Plot of the experimentally determined flux recorded at the SD station, compared with that provided by the W61 wiggler previously installed at the MS beamline. The data have only been corrected for the Pilatus $100 \mathrm{k}$ sensitivity. For each data point the harmonic number at which it was recorded is given. In the inset the beam focus at the SD station at $9 \mathrm{keV}$ is shown. The axes are in micrometres. 


\section{Beamline layout}

The positions of the most important beamline components are listed in Table 4, and shown schematically as a block diagram in Fig. 6. Most of these, in particular the optics between 20 and $31 \mathrm{~m}$, are described below in detail. It is briefly mentioned here that, because the tomography station was removed from the beamline in 2007, an aspect of the upgrade program was to move the powder diffractometer $3.5 \mathrm{~m}$ upstream, while the focusing optics were moved approximately $70 \mathrm{~cm}$ downstream, compared with the former configuration. This means that it is now possible to demagnify the photon beam at PD to 0.6 , which is beneficial for certain experimental set-ups such as those using diamond-anvil cells, or in micro-Laue experiments.

The surface diffractometer has also been moved $70 \mathrm{~cm}$ upstream in the second hutch. Although this has only a marginal impact on the demagnification of the focal spot size, it does allow the possibility of increasing the sampledetector distance to over $2 \mathrm{~m}$. As described in $\$ 9.2 .1$, this becomes important for future possible experiments exploiting coherence, such as coherent X-ray diffraction imaging (CXDI).

\section{The front-end}

The most important front-end components are listed in Table 4, from the mask at $8.245 \mathrm{~m}$ to the CVD diamond window at $17.851 \mathrm{~m}$. The two-blade X-ray beam-position monitors (XBPMs) allow one to monitor both the height and angle of the white X-ray beam produced by U14. Downstream from these are two water-cooled diaphragms which intercept the soft X-ray outer cone of the synchrotron beam. The

Table 4
Positions of important beamline components with respect to the centre of the U14 undulator.

\begin{tabular}{|c|c|c|}
\hline Component & Distance $(\mathrm{m})$ & Comment \\
\hline U14 CPMU & 0 & Power output $=3000 \mathrm{~W}$ \\
\hline Front-end mask & 8.245 & Protection for XBPMs \\
\hline XBPM1 & 8.580 & Blade (white) beam-position monitor \\
\hline XВРM2 & 11.624 & Blade (white) beam-position monitor \\
\hline Diaphragm 1 & 12.510 & $\begin{array}{l}\text { Water cooled; } 4.9 \mathrm{~mm} \times 2.0 \mathrm{~mm} ; 390 \mu \mathrm{rad} \times 160 \mu \mathrm{rad} \\
\quad \text { transmitted power }=1090 \mathrm{~W}\end{array}$ \\
\hline Diaphragm 2 & 17.054 & $\begin{array}{l}\text { Water cooled; } 4.6 \mathrm{~mm} \times 1.2 \mathrm{~mm} ; 270 \mu \mathrm{rad} \times 70 \mu \mathrm{rad} \\
\quad \text { transmitted power }=334 \mathrm{~W}\end{array}$ \\
\hline CVD diamond filter & 17.636 & $120 \mu \mathrm{m}$ thick, water cooled; absorbs soft X-rays \\
\hline CVD diamond window & 17.851 & $\begin{array}{l}80 \mu \mathrm{m} \text { thick, water cooled; vacuum protection for front-end; } \\
\text { transmitted power }=157 \mathrm{~W}\end{array}$ \\
\hline Diamond filter set & 18.855 & $0,50,100,200,400 \mu \mathrm{m}$ thick; water cooled \\
\hline Si filter set & 18.980 & $0,100,200,400,800 \mu \mathrm{m}$ thick; water cooled \\
\hline Beam-defining slits & $19.169-19.319$ & Tungsten, water cooled; accuracy $= \pm 1 \mu \mathrm{m}$ \\
\hline Quadrant BPM 1 & 19.390 & $9.6 \mathrm{~mm} \times 3.6 \mathrm{~mm}$, suitable for pink beam \\
\hline DCM crystal 1 & 20.142 & Centre of $\mathrm{Si}(111)$, liquid- $\mathrm{N}_{2}$ cooled \\
\hline DCM crystal 2 & $20.168-20.367$ & $\begin{array}{l}\text { Centre of } \mathrm{Si}(111) \text {; sagittal focusing system (Schulze } \text { et al., } \\
\text { 1998) }\end{array}$ \\
\hline Quadrant BPM 2 & 20.690 & $9.6 \mathrm{~mm} \times 3.6 \mathrm{~mm}$, not suitable for pink beam \\
\hline Mirror 1 & 21.514 & Centre of Si flat mirror, faces down; adjustable mirror tilt \\
\hline Mirror 2 & 22.214 & $\begin{array}{l}\text { Centre of Si-bendable mirror for vertical focusing, faces up; } \\
\text { adjustable mirror tilt }\end{array}$ \\
\hline $\mathrm{Si}_{3} \mathrm{~N}_{4}$ window & 23.053 & $\begin{array}{l}1 \mu \mathrm{m} \text { thick; two apertures of mm diameter for pink beam } \\
\text { (height }=1400 \mathrm{~mm} \text { ) and monochromatic beam (height }= \\
1420 \mathrm{~mm}) \text {; separates UHV from low-quality vacuum } \\
\text { upstream }\end{array}$ \\
\hline Quadrant BPM 3 & 23.326 & $9.6 \mathrm{~mm} \times 3.6 \mathrm{~mm}$, not suitable for pink beam \\
\hline Bremsstrahlung blocker & 30.485 & $\begin{array}{l}\text { Protection against Bremsstrahlung originating in the storage } \\
\quad \text { ring; } 30 \mathrm{~mm} \mathrm{Cu}+150 \mathrm{~mm} \mathrm{~W}\end{array}$ \\
\hline Powder diffractometer & 32.797 & Experimental hutch 1, demagnification 0.6 \\
\hline Surface diffractometer & 40.087 & Experimental hutch 2, demagnification 0.98 \\
\hline
\end{tabular}

transmitted power through the second (narrower) diaphragm is $334 \mathrm{~W}$.

Approximately half of this transmitted power lies in the soft $\mathrm{X}$-ray regime below approximately $5 \mathrm{keV}$, which interacts very strongly with condensed matter and must therefore be removed to protect the $\mathrm{X}$-ray optical elements. This is achieved with a combination of a $120 \mu \mathrm{m}$-thick water-cooled diamond filter, and an $80 \mu \mathrm{m}$-thick water-cooled diamond window, after which the transmitted power is $157 \mathrm{~W}$. The transmission spectrum of $236 \mu \mathrm{m}$ of diamond is shown in Fig. 4(b). The additional $36 \mu \mathrm{m}$ of diamond is included in order to account for the three quadrant CVD-diamond beamposition monitors (qBPMs) permanently positioned in the beam in the optics hutch (Schulze-Briese et al., 2001).

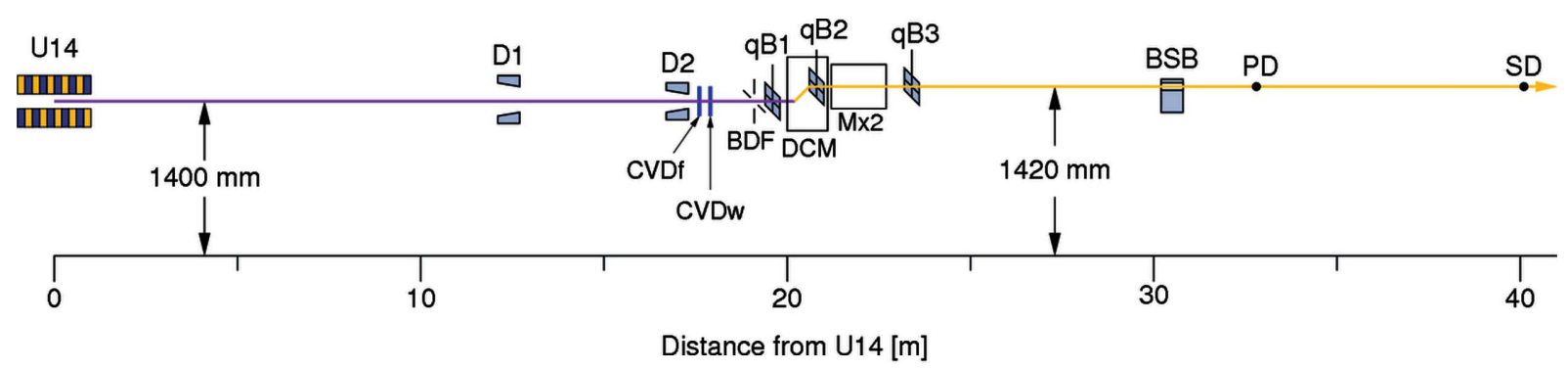

Figure 6

Block diagram of the positions of the most important beamline components. The white/pink beam is shown in magenta, the monochromatic beam in yellow. U14 = U14 undulator; D1 = diaphragm 1; D2 = diaphragm 2; CVDf = CVD filter; CVDw = CVD window; BDF = beam-defining slits; qB1 (2,3) = qBPM 1(2,3); $\mathrm{DCM}=$ double-crystal monochromator; $\mathrm{Mx} 2=$ double mirror chamber; $\mathrm{BSB}=\mathrm{Bremsstrahlung}$ blocker; PD $=$ centre of powder diffractometer; $\mathrm{SD}=$ centre of surface diffractometer. 


\section{Optics design concept}

\subsection{Choice of configuration}

Two mirrors are necessary to sufficiently suppress harmonic contamination (Patterson et al., 2005). The previous optics configuration with W61 was collimating-mirror-DCMfocusing-mirror. As such, the first mirror (1 m long) absorbed as much as $1200 \mathrm{~W}$ of power from the wiggler source and therefore required water cooling (Patterson et al., 2005). There are some important advantages in replacing this set-up with one in which the two mirrors are downstream of the DCM (i.e. DCM-mirror-mirror, see Fig. 7). These include not needing to cool the first mirror, plus the option of using only one of the mirrors for experiments which profit from using as few optical elements as possible, such as CXDI. Lastly, in such a configuration, both mirrors can be housed in the same vacuum vessel, making the design more compact and cheaper.

In reaching a decision as to whether such a change of configuration should be adopted, the relative sizes of the natural vertical divergence (FWHM) of the undulator radiation and the Darwin width of the DCM Si(111) crystals had to be compared. The standard deviation of the undulator radiation perpendicular to the orbital plane is given by

$$
\sigma_{\mathrm{v}}=1 /(n N)^{1 / 2} \gamma
$$

and the FWHM of the beam in this direction is therefore

$$
\mathrm{FWHM}_{\mathrm{v}}=\left(\frac{8 \ln 2}{n N \gamma^{2}}\right)^{1 / 2}
$$

This is plotted in Fig. 8 along with the theoretical Darwin widths of $\mathrm{Si}(111)$ for selected energies. If one makes the simplification that the Darwin curve approximates a top-hat profile of width $2 W_{\mathrm{D}}$, the fraction $f_{\mathrm{D}}$ of the beam diffracted by the $\mathrm{Si}(111)$ crystal is given by

$$
f_{\mathrm{D}}=\operatorname{erf}\left(\frac{W_{\mathrm{D}}}{\sqrt{2} \sigma_{\mathrm{v}}}\right) .
$$

This is also shown in Fig. 8. Where the two angles overlap (approximately $16 \mathrm{keV}$ ), one FWHM of the undulator radia-

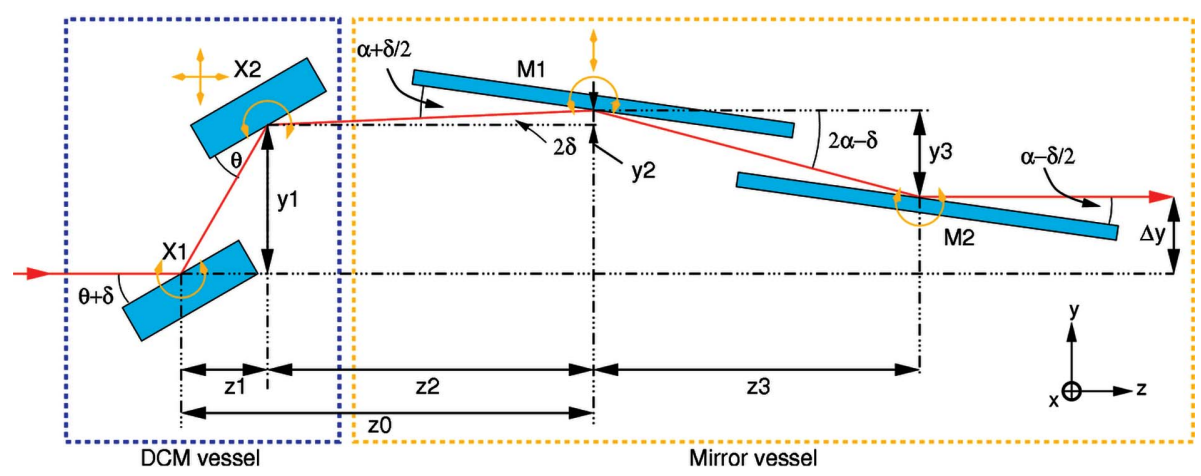

Figure 7

Schematic figure of the optics set-up. Movements required of each of the four components (crystals $\mathrm{X} 1$ and $\mathrm{X} 2$, and mirrors M1 and M2) are shown by yellow arrows. The relevant dimensions used in calculating the optics elements' positions are also given. The beam offset after exiting the mirror chamber is $\Delta y=20 \mathrm{~mm}$.

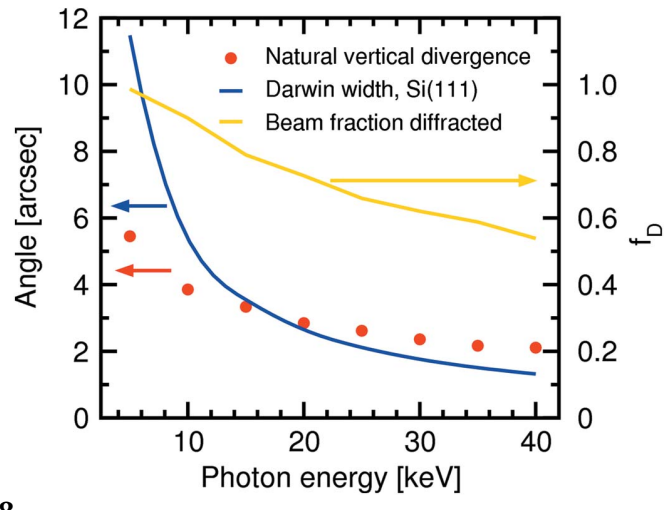

Figure 8

Plot of the theoretical Darwin width and the natural vertical divergence of the monochromatic undulator radiation as a function of photon energy. Also shown is $f_{\mathrm{D}}$, the beam fraction that lies within the Darwin width and is consequently diffracted.

tion is intercepted by the Darwin curve, that is, $76 \%$ of the flux. At the highest desired energy of $40 \mathrm{keV}$, almost $50 \%$ of the beam intensity is lost. Nonetheless, because the large majority of experiments are conducted at photon energies below $25 \mathrm{keV}$, the advantages of this new configuration were deemed to outweigh the loss of flux at higher energies.

\subsection{Detailed geometry}

The fixed-exit DCM design is similar to those already used at the protein crystallography beamline PX1 (Schulze et al., 1998) and the computed tomography beamline TOMCAT (Stampanoni et al., 2007), both at the SLS. The DCM was designed and built by CINEL s.r.l., Padova, Italy (http:// www.cinel.com). Both $\mathrm{Si}(111)$ crystals are mounted on their own goniometer. The second crystal can be bent sagittally to provide horizontal focusing (Schulze et al., 1998) (Fig. 9). A crucial difference, however, between the new optics design at MS and those at PX1 and TOMCAT is the beam offset (i.e. the difference in height between the incoming white beam from the undulator and the outgoing monochromatic beam). At PX1, this is $+50 \mathrm{~mm}$. Because at the MS beamline, we wish to access photon energies as high as $40 \mathrm{keV}$; having a fixed-exit beam with this offset would require the second crystal (X2) to move by approximately $600 \mathrm{~mm}$ in the beam-propagation direction $(z)$, setting unreasonable specifications on the linear-motion stage for $\mathrm{X} 2$ with regards to parasitic motions, and also increase the volume of the monochromator vacuum chamber by a significant fraction. Radiation dosage studies of the Bremsstrahlung radiation at different offsets were performed, from which an offset of $+20 \mathrm{~mm}$ was deemed to be well within the safety limits and meant that the maximum $z$ translation of $\mathrm{X} 2$ is reduced to approximately $200 \mathrm{~mm}$. 
(a)

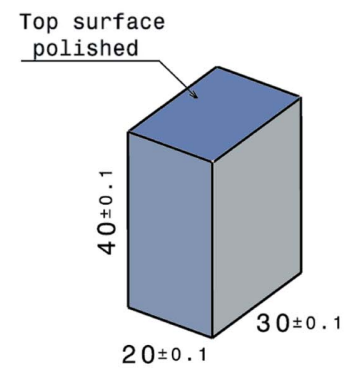

(b)

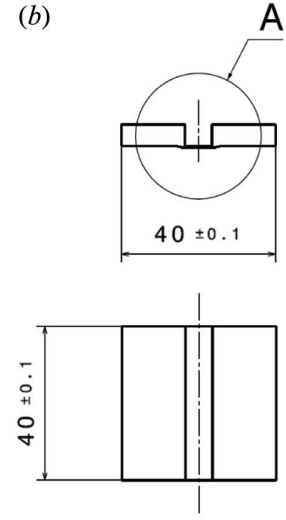

(c)

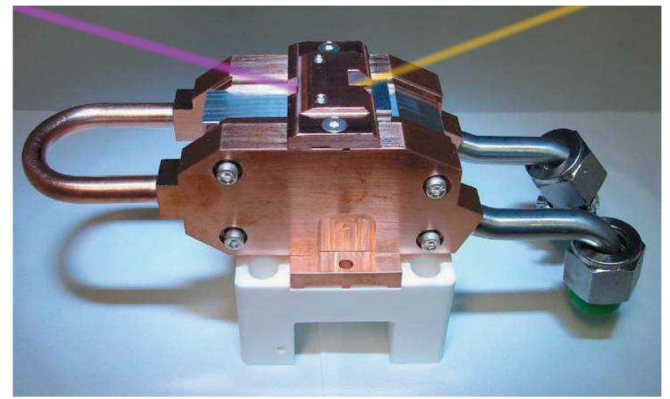

A
Detail A

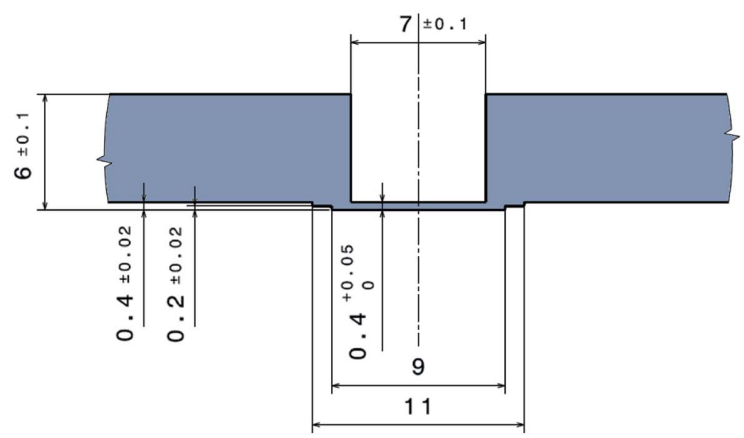

(d)

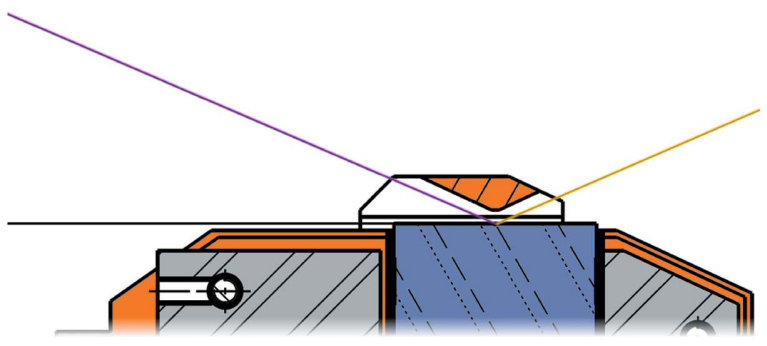

Figure 9

Dimensions of $(a)$ crystal $1(\mathrm{X} 1)$, and $(b)$ crystal $2(\mathrm{X} 2)$ in the DCM, given in millimetres. $(c) \mathrm{X} 1$ mounted in the liquid- $\mathrm{N}_{2}$-cooling housing. The 'white beam' impinges from the left, while the monochromatic beam (here, yellow) emerges on the right. $(d)$ The crystal surface itself is obscured by a copper 'cap' used to protect it from long-term deposits originating from residual-gas carbon-containing species, by limiting the solid angle to which the surface is exposed. Materials: orange $=\mathrm{Cu}$; grey $=\mathrm{Al}$; blue $=\mathrm{Si}$.

This smaller offset compared with that of the PX1 beamline meant, however, that we could not take a carbon copy of the two crystal designs used in the PX1 monochromator (Schulze et al., 1998). The reason for this is that at low photon energies the crystals and their holders would overlap vertically, both occluding the beam and increasing the danger of crashing the crystals into each other when changing the photon energy. As calculated in this section, the vertical separation of X1 and X2 at $5 \mathrm{keV}$ is only approximately $25 \mathrm{~mm}$ (see Fig. 10 below), too small to be able to use the same model of the PX1 beamline. The design concept, however, could be preserved, requiring only that the dimensions of $\mathrm{X} 1$ and $\mathrm{X} 2$ be scaled down. The most important design parameter was that the ratio of the length to width of the thinned bendable section of X2 $(0.4 \mathrm{~mm}$ thick, used for sagittal focusing) be approximately 6 or greater to avoid anticlastic distortions [see Fig. 9(b)].

Another novel feature is shown in Figs. $9(c)$ and $9(d)$. It is well known that the coherent wavefront of quasimonochromatic synchrotron radiation can degrade over time because of the build-up of carbonaceous deposits on X-ray optics surfaces (especially on those exposed to high-intensity polychromatic beams), due to the cracking of carbon-containing species in the residual gas within the vacuum chamber (Chauvet et al., 2011). Considerable effort was invested to optimize the DCM vacuum without incurring unacceptable costs associated with ultrahigh-vacuum (UHV) motors and motion stages. An ultimate pressure better than $10^{-8}$ mbar was specified. This was met by CINEL.
However, the effective incident flux of residual-gas species can be further reduced by an order of magnitude by limiting the solid angle 'seen' by the optics. This was achieved on X1 [on which the high-power white beam is incident, Fig. 9(c)] by installing a copper 'cap' which only opens up to an angle of approximately $25^{\circ}$ perpendicular to the crystal surface (Fig. 9d). The opening solid angle of the cap is approximately $1 \mathrm{sr}$, or $16 \%$ of an unprotected surface. The first $\mathrm{Si}(111)$ crystal $\mathrm{X} 1$ and thus also the cap are maintained at liquid-nitrogen temperatures, and the latter therefore acts as a sacrificial cryopump, protecting X1. After more than one year of operation, no sign of a burn spot could be seen.

A schematic diagram of the DCM and mirrors' configuration is shown in Fig. 7. The second Si crystal X2 is at room temperature. Because of the temperature difference of the two crystals $(\Delta T \simeq 200 \mathrm{~K})$, they also have marginally different Bragg angles, due to the thermal contraction of silicon in $\mathrm{X} 1$.

In order to maintain a constant height for the exit beam after M2 (+20 mm relative to the incoming beam) for all photon energies, the second crystal must be translated both vertically $(y)$ and horizontally $(z)$, while the first mirror M1 must be translated vertically. In addition, the mirror angles are adjusted with photon energy, in order to minimize harmonic content. Both mirrors have three regions: bare silicon, Rhcoating and Pt-coating. The first mirror is kept flat, while the second mirror M2 can be bent to provide vertical (meridional) focusing. 
The thermal expansion coefficient of silicon changes almost linearly between 77 and 300 K (Slack \& Bartram, 1975) such that

$$
\Delta l / l=-2.55 \times 10^{-4}
$$

as one cools from 300 to $77 \mathrm{~K}$.

We let the Bragg angle at $300 \mathrm{~K}$ be $\theta$ for the roomtemperature crystal $\mathrm{X} 2$, and that for the same photon energy of $\mathrm{X} 1$ be $\theta+\delta$. Starting from Bragg's law, it is trivial to demonstrate that

$$
\delta \simeq(\Delta l / l) \tan \theta=2.55 \times 10^{-4} \tan \theta,
$$

where $\delta \ll 1$ is given in radians.

The consequence of this is that the beam is tilted upwards by an angle $2 \delta$ after $\mathrm{X} 2$ (see Fig. 7). This is compensated by tilting the first mirror to an angle of $\alpha+\delta / 2$ relative to the beam and the second mirror to $\alpha-\delta / 2$, so that the exiting beam is again horizontal. Note that $\delta / 2 \alpha \simeq 0.01$ and hence this readjustment has no significant impact on the reflectivity of the mirrors.

The two mirrors were fabricated by WinlightX, Pertuis, France (http://www.winlightx.com) from single-crystal silicon instead of the more conventional material, fused silica. The main reason for this choice was the fact that the thermal conductivity of silicon is 100 times greater than that for silica, thereby minimizing the formation of thermal bumps when operating in pink-beam mode (i.e. bypassing the DCM) for possible future micro-Laue experiments. The mirrors' reflecting surfaces consisted of $7 \mathrm{~mm}$-wide stripes of $\mathrm{Rh}$ coating, bare $\mathrm{Si}$, and $\mathrm{Pt}$ coating (coating thickness of $55 \mathrm{~nm}$ and $37.5 \mathrm{~nm}$, respectively), separated from each other by $4.5 \mathrm{~mm}$. The useful mirror length was $400 \mathrm{~mm}$ and the mirror cross section $40 \mathrm{~mm} \times 40 \mathrm{~mm}$. The tangential slope error was specified to be better than $0.30 \mu \mathrm{rad}$ (RMS). Each stripe is accessed by translation of the entire mirror chamber in the $x$ direction by $\pm 11.5 \mathrm{~mm}$ (see Fig. 7).

The critical angle for total external reflection, $\alpha_{\mathrm{c}}$, is inversely proportional to the photon energy, and proportional to the square-root of the electron density. As is often observed, the actual densities of the sputtered $\mathrm{Rh}$ - and Pt-coating stripes are marginally less than for bulk material. These were accurately determined by recording the reflectivity curves of test pieces prepared in the same sputter-deposition run used to make the mirror coatings themselves, and at three different photon energies. The $\mathrm{Rh}$ - and Pt-coatings were found to be

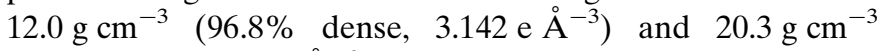

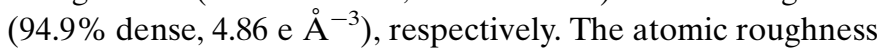
could also be fit, and yielded $0.25 \mathrm{~nm}$ for the $\mathrm{Rh}$ surface and $0.50 \mathrm{~nm}$ for Pt.

The mirrors should be tilted to close to the critical angle, in order to suppress harmonic contamination as much as possible. However, one should avoid getting too close to $\alpha_{\mathrm{c}}$, as the drop in reflectivity at the critical angle is not infinitely abrupt. We therefore chose a value of $85 \%$ of $\alpha_{c}$. The reflectivity at the lower and upper limits of the energy ranges used for each stripe is given in Table 5.

We express this set angle in convenient units as
Table 5

Reflectivity $R$ at $85 \%$ of the critical angle $\alpha_{\mathrm{c}}$ for the three stripes of the two mirrors at their lower and upper limits of the photon energy (given in $\mathrm{keV}$ ).

\begin{tabular}{lll}
\hline Material & $E_{\min } / R$ & $E_{\max } / R$ \\
\hline $\mathrm{Si}$ & $5 / 0.835$ & $10 / 0.950$ \\
$\mathrm{Rh}$ & $10 / 0.833$ & $20 / 0.943$ \\
$\mathrm{Pt}$ & $20 / 0.760$ & $40 / 0.931$ \\
\hline
\end{tabular}

$$
\alpha_{\text {set }}=1.80863 \sqrt{\rho} / E
$$

where $\alpha_{\text {set }}$ is in degrees, $\rho$ is the electron density in e $\AA^{-3}$, and the photon energy $E$ is in $\mathrm{keV}$. This yields

$$
\begin{aligned}
& \alpha_{\text {set }}^{\mathrm{Si}}=1.5078 / E, \\
& \alpha_{\text {set }}^{\mathrm{Rh}}=3.2059 / E, \\
& \alpha_{\text {set }}^{\mathrm{Pt}}=3.9872 / E .
\end{aligned}
$$

Referring to Fig. 7, we see there are some constants to the optics geometry. These are:

(i) The vertical offset of the monochromatic beam emerging from the optics compared with that of the incoming pink beam, $\Delta y=y 1+y 2-y 3=+20 \mathrm{~mm}$.

(ii) The horizontal distance between the centres of M1 and $\mathrm{M} 2, z 3=700 \mathrm{~mm}$.

(iii) The horizontal distance between the centres of $\mathrm{X} 1$ and M1, $z 0=z 1+z 2=1372 \mathrm{~mm}$.

We now determine expressions for the positions and angles of the four optical components. First, the Bragg angle of X2 is simply

$$
\theta=\arcsin (1.977066 / E[\mathrm{keV}])
$$

From Fig. 7,

$$
\begin{gathered}
y 1 / z 1=\tan (2 \theta+2 \delta), \\
y 2 / z 2=y 2 /(z 0-z 1)=\tan 2 \delta, \\
y 3 / z 3=\tan (2 \alpha-\delta) .
\end{gathered}
$$

Remembering that $\Delta y=y 1+y 2-y 3$ leads to

$$
\begin{aligned}
\Delta y & =z 1 \tan (2 \theta+2 \delta)+(z 0-z 1) \tan 2 \delta-z 3 \tan (2 \alpha-\delta) \\
\Rightarrow z 1 & =\frac{\Delta y-z 0 \tan 2 \delta+z 3 \tan (2 \alpha-\delta)}{[\tan (2 \theta+2 \delta)-\tan 2 \delta]} .
\end{aligned}
$$

From this, we directly obtain the horizontal distance between the centres of $\mathrm{X} 2$ and $\mathrm{M} 1, z 2=z 0-z 1$, from which $y 1, y 2$ and y3 directly follow.

The crystal and mirror positions as a function of photon energy for the three mirror coatings are provided in Fig. 10, with the coating densities given above. The limits of the positions at the lowest $(5 \mathrm{keV}$, using $\mathrm{Si}$ reflection on the mirrors) and highest energies (40 keV, using $\mathrm{Pt})$ are shown in Fig. 11. 


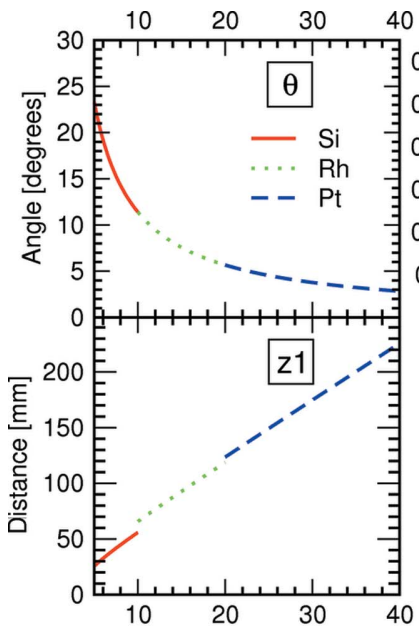

Figure 10

Positions and angles of the optics components as a function of photon energy for the three different mirror-stripe settings.

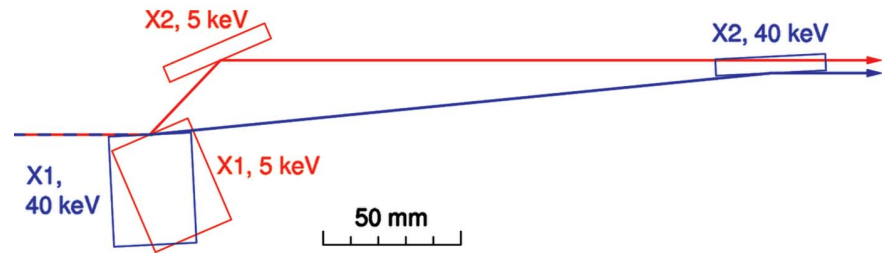

Figure 11

True-scale positions of X1 and X2 at 5 and $40 \mathrm{keV}$, using the Si and Pt stripes on the mirrors, respectively.

\section{Performance}

\subsection{DCM energy resolution}

Initial calibration of the DCM Bragg angles, plus the DCM resolving power, were tested using an unfocused beam by recording the $\mathrm{Cu} K$-edge, which has a well studied pre-edge feature at $8.9813 \mathrm{keV}$. The raw spectrum, only corrected for the small calibration offset in the Bragg angle, is shown in Fig. 12(a), whereby the pre-edge feature is highlighted with an asterisk. The separation in the valley and peak energies of the pre-edge is $1.7 \mathrm{eV}$, or $1.9 \times 10^{-4}$ of the edge energy. This high visibility demonstrates that the DCM energy resolution $\Delta E / E$ is at the theoretical value of approximately $1.4 \times 10^{-4}$.

The calibration offsets for the Bragg angles of X1 and X2 determined from the $\mathrm{Cu}$-edge XANES spectrum are only constant if there is insignificant parasitic pitch associated with the relatively large horizontal movement of X2 between low and high photon energies (see Figs. 10 and 11). A second Pdedge XANES spectrum was recorded at $24.35 \mathrm{keV}$ (Fig. 12b), from which it was observed that $\theta$ was accurate to within $5 \times$ $10^{-5}$ degrees, and that therefore the absolute accuracy of the set energy lay within the Darwin width of the crystals.

Once the DCM has thermally stabilized after initial cooling of the first crystal to $77 \mathrm{~K}$, the photon energy remains constant to better than $0.3 \mathrm{eV}$ (one part in $10^{5}$ ), as was demonstrated by recording at $25 \mathrm{keV}$ a silicon powder pattern every $45 \mathrm{~s}$ over $10 \mathrm{~h}$.

\subsection{Focusing elements}

7.2.1. Primary optics. As mentioned earlier, vertical focusing is achieved using a flexural hinge-based mirror bender, similar to systems described previously (Rossetti et al., 2002). The $\mathrm{X}$-ray beam is focused in the horizontal plane by sagittally bending the second DCM crystal X2 (Schulze et al., 1998).

From the lensmaker equation

$$
\frac{1}{f}=\frac{1}{p}+\frac{1}{q},
$$

whereby $p$ is the source-lens distance and $q$ is the lens-image distance, we can establish the required focal lengths for focusing at the PD station, at the SD station and for parallel beam (Willmott, 2011). For horizontal focusing, $p_{\mathrm{h}} \simeq$ $20.268 \mathrm{~m}$ (the midpoint of the horizontal translation of X2; see Table 4), while, in the vertical plane, $p_{\mathrm{v}}=22.214 \mathrm{~m}$, the centre of mirror 2. For focusing at the powder diffractometer, $q_{\mathrm{h}, \mathrm{PD}}=$ $12.529 \mathrm{~m}$ and $q_{\mathrm{v}, \mathrm{PD}}=10.583 \mathrm{~m}$, while, at the surface diffractometer, $q_{\mathrm{h}, \mathrm{SD}}=19.819 \mathrm{~m}$ and $q_{\mathrm{v}, \mathrm{SD}}=17.873 \mathrm{~m}$. Table 6 lists in the second and third columns the focal lengths in the vertical and horizontal planes for focusing at the powder

Table 6

Focal lengths (in metres) in the horizontal and vertical planes for three modes of operation: focusing at PD, at SD and parallel beam; plus their associated sagittal bending radii $(\mathrm{cm})$, and meridional bending radii $(\mathrm{km})$ at the two limits of the photon-energy range of the MS beamline.

\begin{tabular}{lrrcrlr}
\hline Mode & \multicolumn{1}{c}{$f_{\mathrm{h}}$} & \multicolumn{1}{c}{$f_{\mathrm{v}}$} & $\begin{array}{l}R_{\mathrm{s}} \\
(5 \mathrm{keV})\end{array}$ & $\begin{array}{l}R_{\mathrm{s}} \\
(40 \mathrm{keV})\end{array}$ & $\begin{array}{l}R_{\mathrm{m}} \\
(5 \mathrm{keV})\end{array}$ & $\begin{array}{l}R_{\mathrm{m}} \\
(40 \mathrm{keV})\end{array}$ \\
\hline PD & 7.743 & 7.168 & 612.34 & 76.54 & 2.723 & 8.240 \\
SD & 10.020 & 9.904 & 792.41 & 99.05 & 3.763 & 11.386 \\
$\|$ beam & 20.268 & 22.214 & 1602.8 & 200.4 & 8.440 & 25.537 \\
\hline
\end{tabular}
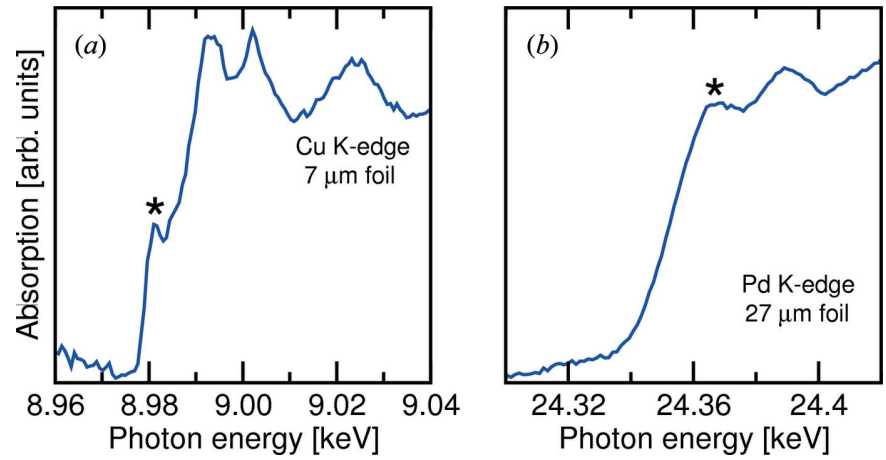

Figure 12

(a) Raw $K$-edge XANES spectrum for metallic copper, recorded at the MS beamline using the unfocused undulator beam. From the width of the pre-edge at $8.9813 \mathrm{keV}$, it was determined that the DCM energy resolution equalled the theoretical value of $1.4 \times 10^{-4}$. (b) Raw $K$-edge XANES spectrum for metallic palladium, recorded to test the reliability of the Bragg angles of the two DCM crystals. 
diffractometer, the surface diffractometer and for parallel beam.

The bending radii $R_{\mathrm{s}}$ and $R_{\mathrm{m}}$ for sagittal focusing using the second DCM crystal and meridional focusing using the second mirror, respectively, are given by

$$
R_{\mathrm{s}}=2 \sin \theta_{i} f_{h}
$$

and

$$
R_{\mathrm{m}}=\frac{2}{\sin \theta_{\mathrm{i}}} f_{\mathrm{v}},
$$

whereby $\theta_{\mathrm{i}}$ is the incident angle of the X-ray beam on the optical element and is equal to the Bragg angle $\theta$ in the case of sagittal focusing using $\mathrm{X} 2$; and equal to the mirror incident angle $\alpha$ for vertical focusing using M2. Both $\theta$ and $\alpha$ are energy dependent. The limiting values for 5 and $40 \mathrm{keV}$ are listed in Table 6 . The change in the bending radii as the beam focus is adjusted between 30 and $43 \mathrm{~m}$ from the centre of U14 is shown in Fig. 13.

We now consider the necessary range of motion and resolution of the actuators used to bend X2 and M2. We begin by assuming cylindrical focusing. The pertinent geometries are sketched in Fig. 14. We begin with the simpler configuration of the mirror bender (Fig. 14b). From Pythagoras' theorem,

$$
R_{\mathrm{m}}^{2}-\left(R_{\mathrm{m}}-y_{\mathrm{m}}\right)^{2}=L_{\mathrm{m}}^{2},
$$

from which one obtains

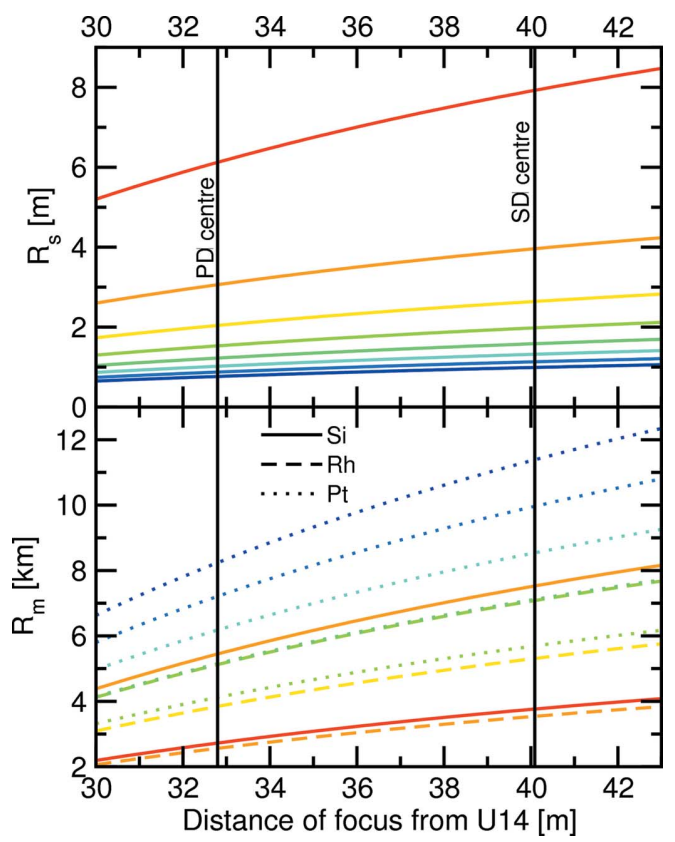

Figure 13

Change in the sagittal and meridional bending radii as the focus is adjusted between 30 and $43 \mathrm{~m}$. The colours of the curves change gradually in $5 \mathrm{keV}$ steps between $5 \mathrm{keV}$ (red) and $40 \mathrm{keV}$ (blue). Because the meridional focus depends on the mirror incident angle, which in turn depends on which stripe is being used ( $\mathrm{Si}, \mathrm{Rh}$ or $\mathrm{Pt})$, these are distinguished by the curves being solid, dashed and dotted, respectively. The positions of the powder diffractometer and surface diffractometer centres are also marked.

$$
y_{\mathrm{m}}=L_{\mathrm{m}}^{2} / 2 R_{\mathrm{m}}
$$

under the valid assumption that $y_{\mathrm{m}} / R_{\mathrm{m}} \ll 1$. Both mirrors in the new optics set-up have a usable length $2 L_{\mathrm{m}}=400 \mathrm{~mm}$. The maximum value for $y_{\mathrm{m}}$ is thus at the minimum bending radius, which is required for focusing $5 \mathrm{keV}$ photons at the PD station $\left(R_{\mathrm{m}}=2.723 \mathrm{~km}\right)$, and therefore $y_{\mathrm{m}}^{\max }=7.34 \mu \mathrm{m}$.

Differentiation of (26) with respect to $R_{\mathrm{m}}$ leads to

$$
\frac{\mathrm{d} y_{\mathrm{m}}}{\mathrm{d} R_{\mathrm{m}}}=\frac{-L_{\mathrm{m}}^{2}}{2 R_{\mathrm{m}}^{2}} .
$$

This tells us the change in $y_{\mathrm{m}}\left(\mathrm{d} y_{\mathrm{m}}\right)$ for a small change in the meridional bending radius $\mathrm{d} R_{\mathrm{m}}$. What interests us more, however, is the amount we need to move the actuator for a given small change in $q$, the position of the focal spot (i.e. $\mathrm{d} q$ ). We differentiate by parts equation (24) with respect to $q$ to obtain

$$
\begin{aligned}
\frac{\mathrm{d} R_{\mathrm{m}}}{\mathrm{d} q} & =\frac{2}{\sin \alpha}\left[\frac{-p q}{(p+q)^{2}}+\frac{p}{p+q}\right] \\
& =\frac{2 p^{2}}{(p+q)^{2} \sin \alpha} .
\end{aligned}
$$

Multiplying (27) with (28), we obtain

$$
\frac{\mathrm{d} y_{\mathrm{m}}}{\mathrm{d} R_{\mathrm{m}}} \frac{\mathrm{d} R_{\mathrm{m}}}{\mathrm{d} q}=\frac{\mathrm{d} y_{\mathrm{m}}}{\mathrm{d} q}=\frac{-1}{\sin \alpha}\left[\frac{L_{\mathrm{m}} p}{R_{\mathrm{m}}(p+q)}\right]^{2} .
$$

Substituting for $R_{\mathrm{m}}$ using (24) leads to

(a)

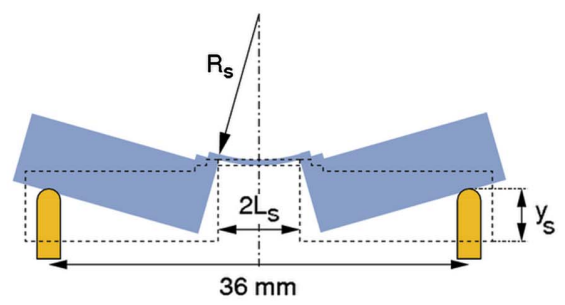

(b)

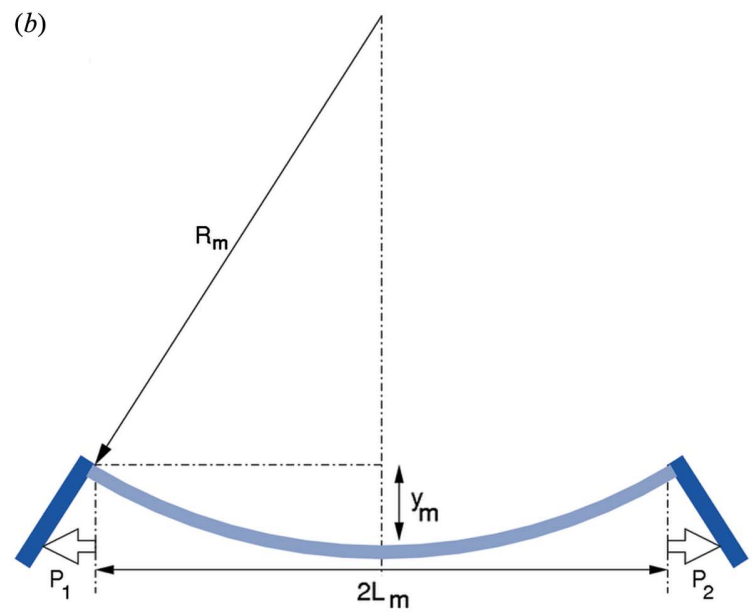

Figure 14

Schematics of $(a)$ the sagittal bender geometry and actuators; and $(b)$ the meridional bender geometry. The mirror is bent by applying outwards forces on Si blades (shown here in dark blue) attached to the mirror ends, which are pushed outwards by equal amounts $P_{1}$ and $P_{2}$. 


$$
\frac{\mathrm{d} y_{\mathrm{m}}}{\mathrm{d} q}=\frac{-L_{\mathrm{m}}^{2} \sin \alpha}{4 q^{2}} .
$$

We want to control the focal position with an accuracy of $10 \mathrm{~cm}$. From (30), the smallest change in $y_{\mathrm{m}}, \mathrm{d} y_{\mathrm{m}}$, is required for large $q$ (i.e. at the surface diffractometer) and for high photon energies (small incident angle $\alpha$ ). Under these conditions, control of $q$ with an accuracy $\mathrm{d} q=10 \mathrm{~cm}$ therefore requires $\mathrm{d} y_{\mathrm{m}}=5.4 \mathrm{~nm}$, approximately 1400 times smaller than $y_{\mathrm{m}}^{\max }$.

Bending mirror 2 was achieved using the flexural hinge system described by Rossetti et al. (2002). Silicon blades, $10 \mathrm{~mm}$-thick, are attached to a rigid clamp system holding the ends of the Si mirror. The bending depth $y_{\mathrm{m}}$ is proportional to the amount the Si blades are pushed outwards $\left(P_{1}\right.$ and $P_{2}$ in Fig. 14). It is hence clear from equation (26) that the meridional radius of the second mirror, $R_{\mathrm{m}}$, is inversely proportional to the positions of the Si-blade actuators. This is demonstrated in Fig. 15 using a long-trace profiler. Driving both motors to push out the blades by $P_{1}=P_{2}=0.5 \mathrm{~mm}$ was shown to induce the minimum bending radius of approximately $2 \mathrm{~km}$.

$\mathrm{X} 2$ is sagittally bent by applying equal forces on four actuators (two pairs, each pair being separated by $36 \mathrm{~mm}$ ). From Fig. 14(a), we see that the height of the four actuators, $y_{\mathrm{s}}$, above that in the flat configuration, is composed of two parts: $y_{\mathrm{s} 1}$, due to the bending of the thinned central part of width $2 L_{\mathrm{s}}=7 \mathrm{~mm}$, and $y_{\mathrm{s} 2}$, produced by the tilting of the rigid blocks. $y_{\mathrm{s} 1}$ is derived in the same manner as $y_{\mathrm{m}}$ in equation (26), so that

$$
y_{\mathrm{s} 1}=L_{\mathrm{s}}^{2} / 2 R_{\mathrm{s}} .
$$

$y_{\mathrm{s} 2}$ can be simply derived by using the principle of similar triangles,

$$
\begin{aligned}
\frac{L_{\mathrm{s}}}{R_{\mathrm{s}}} & =\frac{y_{\mathrm{s} 2}}{\left(36-2 L_{\mathrm{s}}\right) / 2} \\
\Rightarrow y_{\mathrm{s} 2} & =\frac{L_{\mathrm{s}}\left(36-2 L_{\mathrm{s}}\right)}{2 R_{\mathrm{s}}} .
\end{aligned}
$$

Combining (31) and (32) we obtain

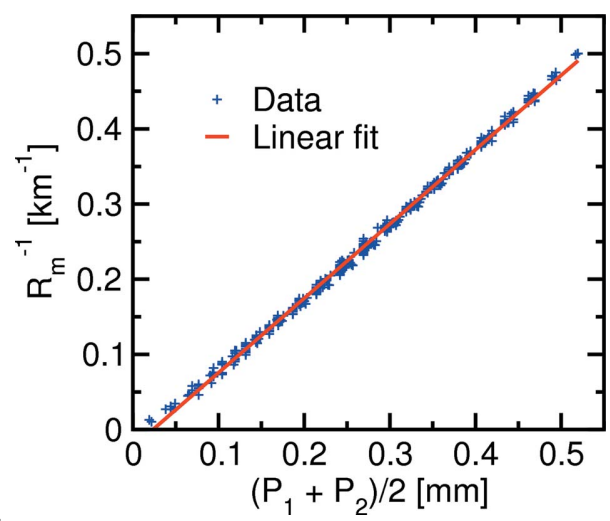

Figure 15

The bending radius of the second mirror measured using a long-trace profiler versus the average of the Si-blade actuator positions $\left(P_{1}+P_{2}\right) / 2$. The best linear fit is also shown.

$$
\begin{aligned}
y_{\mathrm{s}} & =\frac{L_{\mathrm{s}}^{2}}{2 R_{\mathrm{s}}}+\frac{L_{\mathrm{s}}\left(36-2 L_{\mathrm{s}}\right)}{2 R_{\mathrm{s}}} \\
& =\frac{\left(36-L_{\mathrm{s}}\right) L_{\mathrm{s}}}{2 R_{\mathrm{s}}} .
\end{aligned}
$$

The maximum travel of the sagittal actuators is therefore approximately $75 \mu \mathrm{m}$.

Using the same approach as above for meridional focusing, and referring to (33) and (23), leads to

$$
\frac{\mathrm{d} y_{\mathrm{s}}}{\mathrm{d} q}=\frac{\left(L_{\mathrm{s}}-36\right) L_{\mathrm{s}}}{4 q^{2} \sin \theta} .
$$

Because of the larger divergence in the horizontal plane compared with that in the vertical plane, it is unnecessary to control the focal point to better than approximately $1 \mathrm{~m}$. Control of $q$ to this amount thus requires (at low photon energies at the surface station) a minimum step size in $y_{\mathrm{s}}$ of $180 \mathrm{~nm}$. In the present set-up, the pitch of the sagittal actuators is $250 \mu \mathrm{m}$ and the motors have a resolution of 1000 steps per turn, sufficient for all conditions except the lowest-energy experiments at the SD station.

Both mirrors can be dynamically tilted ('pitch' motion), a requirement set by the change in incident angle with photon energy [Fig. 16(a) and equations (14)-(16)]. We specified the tilt accuracy to be equal to or better than $10 \mu \mathrm{m}$ in the vertical position at the SD station. This imposes an accuracy of better than $80 \mathrm{~nm}$ for the linear positions of the $3 \times 2$ linear drives used to tilt the mirrors. These drives, adapted in-house from an earlier design from DESY, Hamburg, were found to have an accuracy of better than $50 \mathrm{~nm}$ when operated in open loop. Under normal operating conditions, the absolute positions were controlled using encoders with $50 \mathrm{~nm}$ resolution. A sketch of the linear drive design is shown in Fig. 16(b).

The best focal spots obtained to date using the primary optics have been $80 \mu \mathrm{m} \times 30 \mu \mathrm{m}$ at the PD station and $130 \mu \mathrm{m}$ $\times 40 \mu \mathrm{m}$ at the SD station.

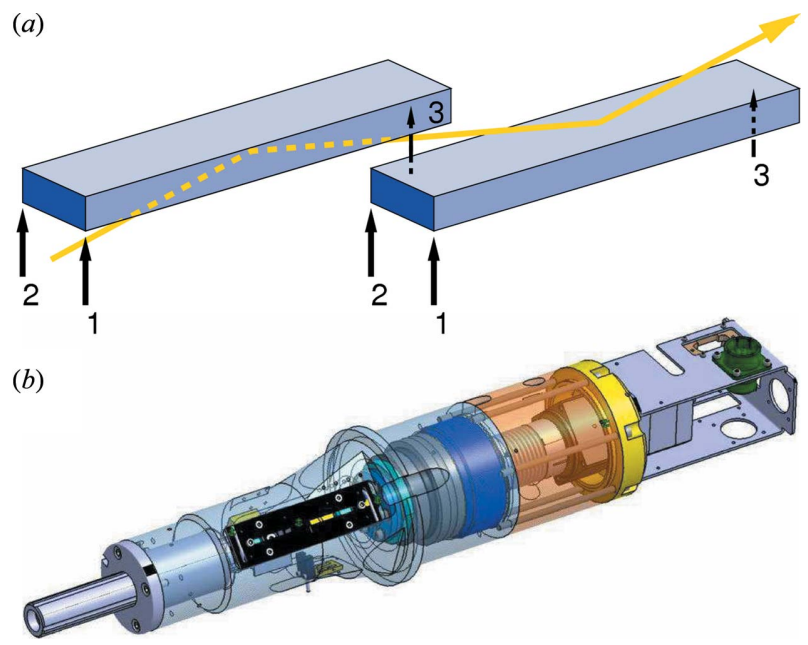

Figure 16

(a) Schematic of the linear drive set-up for tilting and rolling the X-ray mirrors. (b) Detail of the drive design. 


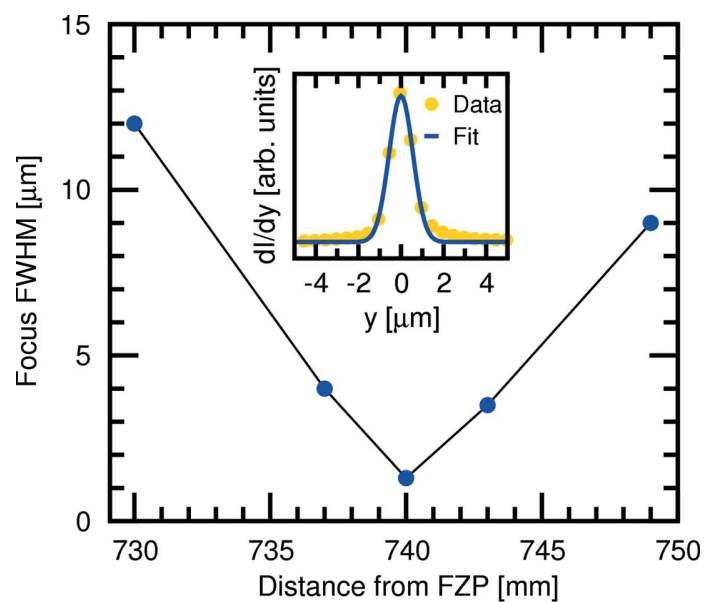

Figure 17

Change in the focused FWHM beam diameter as a function of distance downstream from the FZP. The tightest focus at $740 \mathrm{~mm}$ has a FWHM width of $1.32 \mu \mathrm{m}$ (see inset).

7.2.2. FZP microfocusing. Fresnel zone plates (FZPs) fabricated in-house by the Laboratory of Micro- and Nanotechnology can be employed to demagnify the source by approximately a factor of 50 . The first FZP, made of $0.82 \mu \mathrm{m}$ thick gold Fresnel zones on a $\mathrm{Si}_{3} \mathrm{~N}_{4}$ membrane (outer zone width of $100 \mathrm{~nm}$ and diameter of $1 \mathrm{~mm}$ ) was tested at the SD station using $9 \mathrm{keV}$ radiation. The FZP nominal focal length is $725.9 \mathrm{~mm}$. The source-sample distance at the SD station is $40.087 \mathrm{~m}$ (see Table 4); hence, using the lensmaker's equation, we would expect the tightest focus at a distance of $739.5 \mathrm{~mm}$ downstream from the FZP. The experimentally determined smallest focus was found to be at this position to within $0.5 \mathrm{~mm}$, had a FWHM of $1.32 \mu \mathrm{m}$ (Fig. 17) and transmitted a flux of $5 \times 10^{10}$ photons s ${ }^{-1}$, equating to a flux density of $3.7 \times$ $10^{16}$ photons $\mathrm{s}^{-1} \mathrm{~mm}^{-2}$, approximately 12 times higher than the best focus at SD using only the primary optics. The FWHM beam divergence using this FZP is $1.35 \mathrm{mrad}\left(0.077^{\circ}\right)$.

\section{Endstations}

\subsection{Powder diffraction}

8.1.1. Mythen II microstrip detector. The Mythen II microstrip detector has become the workhorse of the PD station (Bergamaschi et al., 2010) since its installation in 2007. The Mythen detector has recently undergone a significant upgrade, in which a second layer of detector modules was installed. Each layer covers an angle of $120^{\circ}$ and consists of 24 modules. The geometry of this new set-up is shown in Fig. 18. The radii of the circles tangential to the layer surfaces are $761.5 \mathrm{~mm}$ (inner layer, Si-module thickness $320 \mu \mathrm{m}$ ) and $784.45 \mathrm{~mm}$ (outer layer, thickness $450 \mu \mathrm{m}$ ). The modules of both layers are 1280 channels wide (channel separation of $50 \mu \mathrm{m})$ and are repeated every $5^{\circ}$. The outer layer is offset relative to the inner layer by approximately $1.25^{\circ}$, in order to provide a gapless angular response, thereby obviating the need to move the detector to cover the small gaps between modules $\left(0.179^{\circ}\right.$ and $0.320^{\circ}$ for the inner and outer layer,

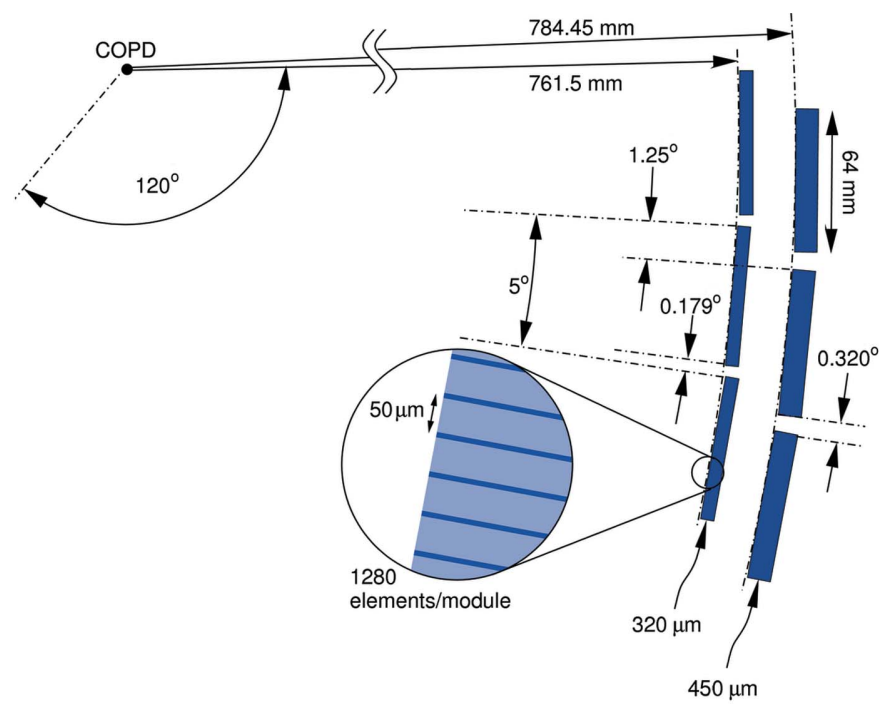

Figure 18

Schematic of the most important geometrical parameters of the upgraded Mythen II detector at the PD station. COPD = centre of the powder diffractometer.

respectively). The minimum readout time (i.e. dead-time when operating in the non-symmetric mode; see below) is $90 \mu$ s for 4-bit low-dynamic-range data.

There are further advantages in having the second layer. Firstly, the thicker outer modules are more sensitive to highenergy X-rays. Secondly, rate corrections for intense (e.g. Bragg-peak) signal can be avoided or at least reduced, since the data on the inner layer can be discarded, because the statistics for the signal from the outer layer are already high.

The intrinsic resolution of Mythen II in $2 \theta$ is $\arctan (0.05 /$ $761.5)=0.00376^{\circ}$. The effective resolution, however, is determined by the sample or illumination size, such that, for example, the resolution of data acquired from a homogeneously illuminated capillary of $200 \mu \mathrm{m}$ diameter is $0.0188^{\circ}$ (Gozzo et al., 2004, 2010). In the large majority of cases, this is more than sufficient for PD applications, be they structural solutions, profile/full-pattern analysis or total scattering. Only in rare cases in which the sample intrinsic line broadening is close to line-profile standards is the resolution using Mythen II inadequate. In particular, Mythen II has been shown to be perfectly sufficient for full structural determination (Bruni et al., 2011). Moreover, the parallel data acquisition over large angular ranges means that $(a)$ time-resolved studies on a millisecond timescale or shorter and $(b)$ the complete exclusion of radiation damage, are possible with Mythen II, which would be impossible using analyzer detectors (Bergamaschi et al., 2010; Fadenberger et al., 2010; Willmott, 2011).

The upgrade of Mythen II has also included operational improvements. It can now be used in a symmetrical configuration $\left( \pm 60^{\circ}\right)$, whereby data acquisition from one half is delayed with respect to the other, for dead-time-free measurements. When operating continuously and with the maximum dynamic range of 24 bits (16 Mcounts), the maximum data acquisition rate for the full $120^{\circ}$ is 20 frames s$^{-1}$. This scales inversely with the number of 


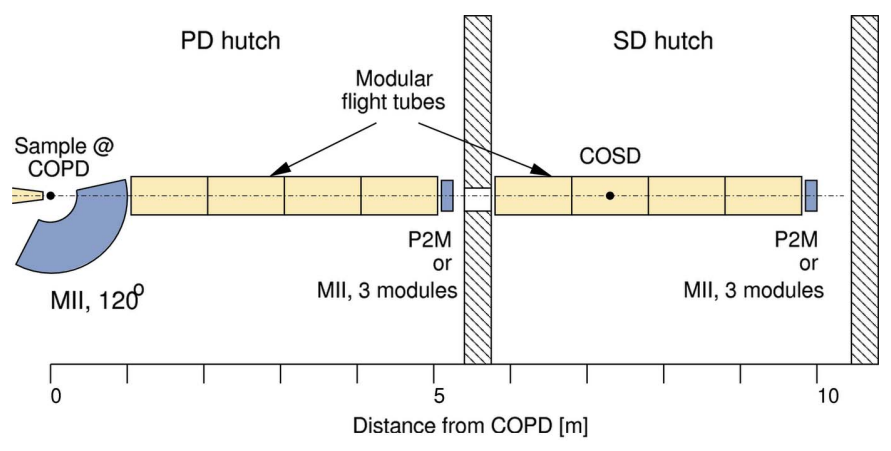

Figure 19

Schematic of the possible set-ups for SAXS/WAXS experiments at the MS beamline. A transparent window in the housing of the $120^{\circ}$ Mythen II detector (MII, $120^{\circ}$, used for WAXS data) reduces the dead region to zero (or the beam stop size) between scattering up and down. The SAXS data can either be recorded in the PD hutch using a Pilatus 2M detector $[\mathrm{P} 2 \mathrm{M}$, area $253 \mathrm{~mm}(\mathrm{~h}) \times 288 \mathrm{~mm}(\mathrm{v})]$ or three Mythen II modules (length $\simeq 192 \mathrm{~mm}$ ). Extension to sample-detector distances as large as $10 \mathrm{~m}$ is possible if the $\mathrm{P} 2 \mathrm{M}$ is placed in the SD hutch. The evacuated or He-filled flight tubes are constructed from modular $1 \mathrm{~m}$-long units. COSD $=$ centre of the surface diffractometer.

modules used and the dynamic range. Lastly, the on-board memory allows for storage of data up to several thousand frames per burst before data must be transferred to file.

8.1.2. SAXS/WAXS facility. A new combined small-angle/ wide-angle X-ray scattering (SAXS/WAXS) set-up has been designed at the MS beamline, with a view to investigating phase changes and catalytic processes on micrometre- and submicrometre-sized powder systems. Fig. 19 shows the possible configurations. WAXS data are recorded using the large Mythen II microstrip detector. A crystalline transparent window at one edge of the WAXS detector housing allows the SAXS signal to propagate with zero dead region on-axis (in reality, the dead region is determined by a beam stop directly in front of the SAXS detector). The sample-SAXS detector distance can be varied, according to the length of the flight tube in between, which consists of modular $1 \mathrm{~m}$ sections. The choice of the SAXS detector depends on the needs of the experiment; the three-module Mythen II detector provides better resolution in reciprocal space, but only in one dimension, and has a smaller maximum scattering vector $Q_{\max }$. Conversely, the Pilatus $2 \mathrm{M}$ detector is $50 \%$ larger and can therefore in principle detect features that are correspondingly smaller, although the largest objects that can be resolved are three times smaller than those studied with the Mythen modules.

The largest scattering vector $Q_{\max }$ and best resolution $\Delta Q$ are listed for both WAXS and SAXS at a photon energy of $10 \mathrm{keV}$ in Table 7.

8.1.3. Other equipment. Several sample environments are available for users at the PD station, listed in Table 8. In addition, a robotic sample changer (Stäubli, model TX60L) allows rapid and remote sample exchange, particularly useful for experiments in which many samples need to be investigated (Fig. 20a). Most of such experiments are performed using the Mythen detector (and not the crystal analyzer), hence it is imperative that the sample capillary is mounted as
Table 7

Largest scattering vectors $Q_{\max }$ and best resolution $\Delta Q$ for different configurations in the SAXS/WAXS set-up at $10 \mathrm{keV}$ (see also Fig. 19).

\begin{tabular}{lll}
\hline Detector/distance $(\mathrm{m})$ & $Q_{\max }\left(\AA^{-1}\right)$ & $\Delta Q\left(\AA^{-1}\right)$ \\
\hline MII, 120 $(\mathrm{WAXS}) / 0.761$ & 8.78 & $1.66 \times 10^{-4}$ \\
$\mathrm{P} 2 \mathrm{M}(\mathrm{SAXS}) / 1.2$ & $1.191 \dagger$ & $7.21 \times 10^{-4}$ \\
MII, 3 modules (SAXS)/1.2 & $0.803 \dagger$ & $2.10 \times 10^{-4}$ \\
P2M (SAXS)/5 & $0.292 \dagger$ & $1.74 \times 10^{-4}$ \\
MII, 3 modules (SAXS)/5 & $0.194 \dagger$ & $5.07 \times 10^{-5}$ \\
P2M (SAXS)/10 & $0.044 \ddagger$ & $8.72 \times 10^{-5}$ \\
MII, 3 modules (SAXS)/5 & $0.044 \ddagger$ & $2.53 \times 10^{-5}$ \\
\hline
\end{tabular}

$\dagger$ Assumes direct beam at edge of detector. $\ddagger$ Limited by the opening angle $(2 \theta=$ $0.50^{\circ}$ ) of aperture between PD and SD hutch $5.6 \mathrm{~m}$ downstream from the centre of the powder diffractometer (COPD)

Table 8

List of available sample environments at the PD station.

\begin{tabular}{lll}
\hline Equipment & Operating range & Comments \\
\hline Janis cryostat & $4.5-300 \mathrm{~K}$ & Capillary samples only \\
Oxford cryojet & $90-500 \mathrm{~K}$ & \\
Heat gun & $300-1300 \mathrm{~K}$ & \\
Stoe furnace & $300-1700 \mathrm{~K}$ & Capillary samples only \\
MRI furnace & $300-2000 \mathrm{~K}$ & Flat-plate samples only \\
Membrane diamond-anvil cell & $0.1-20 \mathrm{GPa}$ & 0.5 mm-diameter culets \\
\hline
\end{tabular}
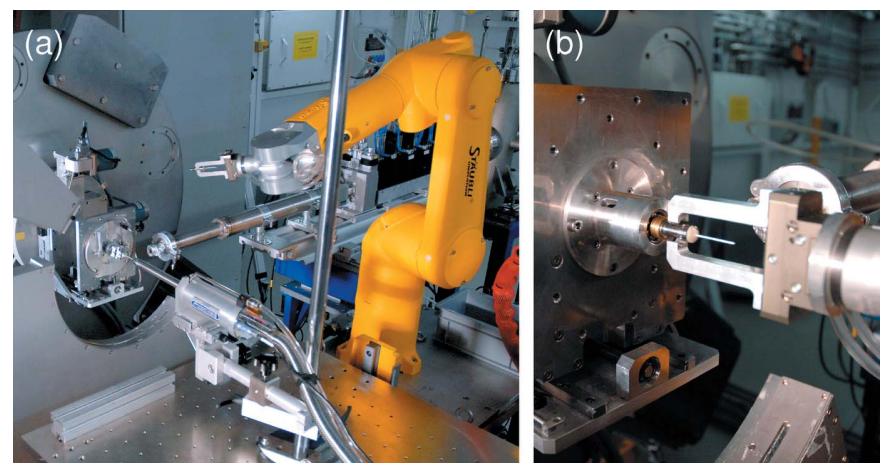

Figure 20

The robotic Stäubli TX60L sample changer at the PD station. (a) The robot arm in relation to the powder diffractometer. $(b)$ Close-up of the sample grabber and magnetic cone mounting system.

coaxially as possible with the diffractometer axis, as the sample holders used by the robot system allow no adjustment of the sample orientation (Fig. 20b). A system is presently under development to simply and rapidly mount capillaries of all diameters concentrically on the magnetic cone sample holders.

\subsection{Surface diffraction}

8.2.1. Detectors. The two-dimensional photon-counting Pilatus 100k detector has been permanently installed at the SD station since 2005 (Schlepütz et al., 2005, 2011). The original graphite-crystal analyzer has been removed. In the present set-up, the sample-detector distance of $1140.8 \mathrm{~mm}$ and pixel size of $172 \mu \mathrm{m} \times 172 \mu \mathrm{m}$ provides an angular resolution of $0.00862^{\circ}\left(\Delta Q=7.6 \times 10^{-4} \AA\right.$ at $\left.10 \mathrm{keV}\right)$. 
At the time of writing, steps are being taken to replace the Pilatus 100k detector with the next-generation 'Eiger 500k' detector (Dinapoli et al., 2010), consisting of $1024(\mathrm{~h}) \times 512$ (v) pixels, each pixel being $75 \mu \mathrm{m} \times 75 \mu \mathrm{m}$ in size. At the same sample-detector distance as presently used, this will provide a resolution of $\Delta Q=3.3 \times 10^{-4} \AA$ at $10 \mathrm{keV}$. As discussed in \$9.2.1, extending the sample-detector distance to $2 \mathrm{~m}$ will enhance the resolution to approximately $1.9 \times 10^{-4} \AA$.

The 'standard' Eiger module is water-cooled. Although this poses no problem for experiments in which the detector is stationary, such as in SAXS, water-cooling is difficult to implement at the surface diffractometer, as the detector module is mounted on the rotatable detector arm. The Eiger module being developed for the SD station is therefore aircooled.

8.2.2. Environmental chambers. For robust sample surfaces, in particular those of many perovskites and related complex metal-oxides, it is only necessary to maintain an oxygen/water partial pressure below approximately $1 \mathrm{mbar}$ in order to avoid radiation damage. For such samples, two very simple and easyto-use systems are available. The first is a $50 \mathrm{~mm}$-diameter $0.5 \mathrm{~mm}$-thick beryllium dome, pumped via a Teflon tube with a turbomolecular pump to approximately $10^{-2}$ mbar. The alternative to this is an $80 \mathrm{~mm}$-diameter $25 \mu \mathrm{m}$-thick thermoformable polyimide dome filled with helium gas. This system has the important advantage over the Be dome that there is essentially no diffuse scattering of the incident and exit beam by the dome walls, and, because the plastic is amorphous, polycrystalline Debye-Scherrer cone signal from the dome is entirely absent.

Recently, a new UHV cryostat has been designed and constructed for SD experiments. The lowest achievable temperature is $13 \mathrm{~K}$. This replaces the previous system, which was designed for bulk single-crystal diffraction experiments and was thus far from optimal for SD experiments. The sample movements in the new design (i.e. rotation of the sample about its surface normal, and fine adjustment of the surface orientation using the hexapod) are decoupled from the vacuum vessel (which is fixed to the optical table on the surface diffractometer) using bellows and a ferrofluid rotary feedthrough (see Fig. 21a). A rod is attached to the vacuum side of
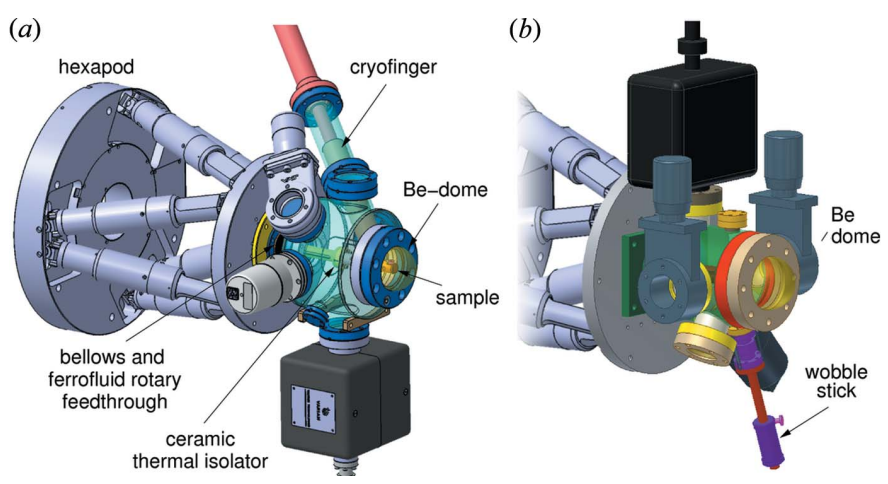

Figure 21

(a) UHV cryostat chamber for experiments in which the sample needs to be cooled to as low as $4.2 \mathrm{~K}$. (b) UHV 'baby chamber' for experiments requiring vacuum below $10^{-9}$ mbar. this feedthrough, which is in turn connected via a ceramic spacer to the cooled sample holder, made from copper. The spacer thereby thermally isolates the sample mount from the rest of the chamber, allowing for rapid thermal equilibration and mechanically stable conditions. The sample holder is connected via copper braids to the Janis ST-400-1 UHV continuous-flow cryostat. As well as cooling, the cryostat can be heated to $500 \mathrm{~K}$. All vacuum seals are UHV-compatible conflat flanges. The ultimate pressure lies in the mid $10^{-10}$ mbar range.

For experiments requiring only the best vacuum conditions, a second 'mini chamber' is available, based on the design of Lee \& Zegenhagen (2006). Samples can be inserted and removed by attaching a vacuum load lock onto a side port and are held using screws that are manipulated using a wobble stick (see Fig. 21b). The ultimate pressure lies in the low $10^{-10}$ mbar range.

Lastly, an in situ thin-film growth chamber using pulsed laser deposition is also available, as described in detail elsewhere (Willmott et al., 2005).

\section{Initial results}

\subsection{Powder diffraction}

Standard Si-PD patterns were recorded at $20 \mathrm{keV}$ using the Mythen II detector, under comparable conditions [with regards to the capillary diameter $(300 \mu \mathrm{m})$, filling fraction, length of capillary exposed, and vertical beam divergence], and using parallelized radiation from the W61 wiggler in 2010 and naturally divergent radiation from the U14 undulator in 2012. In the former the capillary was exposed for $160 \mathrm{~s}$, while in the latter the exposure time was $40 \mathrm{~s}$. The results are compared in Fig. 22. The count rate is approximately eight times larger when using undulator radiation. This corresponds very well to the theoretically expected ratio of the beam crosssections at the powder station, assuming similar total flux from U14 and W61 at this energy.

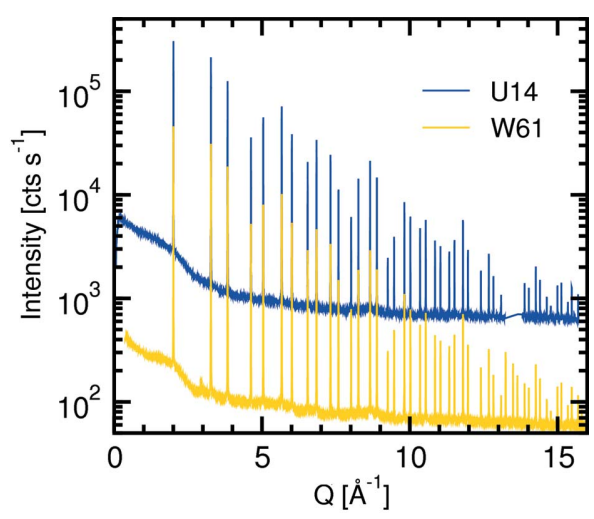

Figure 22

Powder patterns of Si powder recorded using wiggler radiation (gold) and undulator radiation (blue). The approximately factor-of-two larger noise in the latter is due to the fact that the exposure time was four times shorter than in the former. 


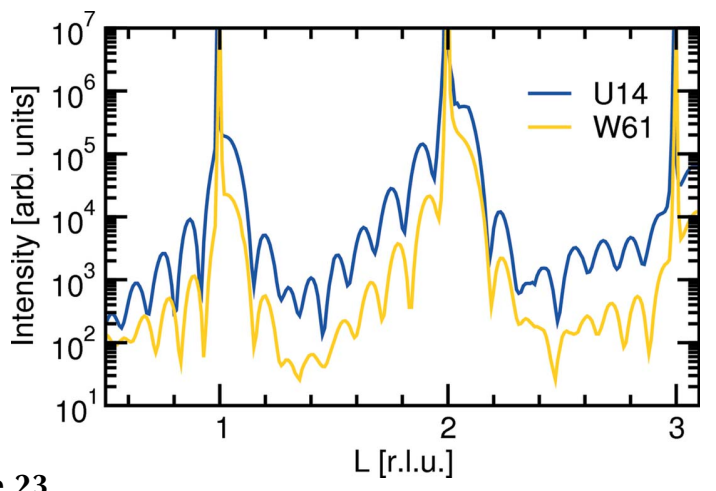

Figure 23

The $(00 l)$ crystal-truncation rod of the same $\mathrm{LaNiO}_{3}$ thin film grown on $\mathrm{SrTiO}_{3}$ using the W61 wiggler and the U14 undulator.

\subsection{Surface diffraction}

A comparison of the $(00 l)$ crystal-truncation rod (CTR) of the same $\mathrm{LaNiO}_{3}$ thin-film sample using wiggler and undulator radiation at $15.5 \mathrm{keV}$ is shown in Fig. 23. A similar increase in signal intensity (after background subtraction) of approximately a factor of ten was obtained. Marginal differences in the details of the CTR profiles can be explained by the footprint of the undulator radiation being approximately a factor of five smaller than that of the wiggler source, plus the fact that the $\mathrm{LaNiO}_{3}$ sample was two years older when the U14-CTR was recorded.

9.2.1. Coherent X-ray diffraction imaging. Coherent X-ray diffraction imaging (CXDI) in the Bragg geometry is an emerging quantitative technique for imaging the internal defect structure of nano- and micro-crystalline objects and materials (Robinson \& Harder, 2009) and extended samples (Robinson et al., 2005; Le Bolloc'h et al., 2005). Phase retrieval and inversion of the oversampled intensity distribution around a Bragg peak (Sayre, 1952) allows one to obtain the electron density and relative displacements (i.e. strain fields) with subångström resolution of the atoms from their ideal bulk-like positions (Pfeifer et al., 2006).

At present the coherence volume available at the MS beamline is of the order of $\xi_{\mathrm{T}, \mathrm{h}}=10 \mu \mathrm{m}, \xi_{\mathrm{T}, \mathrm{v}}=100 \mu \mathrm{m}, \xi_{\mathrm{L}}=$ $1 \mu \mathrm{m}$. The $2+3$ circle surface diffractometer allows sufficient degrees of freedom to access and map in three dimensions individual Bragg reflections. The secondary optic FZP described in $\$ 7.2 .2$ allows micrometre focusing.

At the end of November 2012, the first CXDI experiments were performed at the MS beamline. Two modes of operation were tested: one in which the transmitted coherent flux was selected from the unfocused beam using a coherence defining slit $[20 \mu \mathrm{m}(\mathrm{h}) \times 100 \mu \mathrm{m}(\mathrm{v})]$, the second with the FZP optics described above.

In Fig. 24, the source coherence was confirmed in the diffraction pattern of a $500 \mathrm{~nm}$ gold crystal by the high visibility of the fringes. The example shown was collected at $9 \mathrm{keV}$ using the FZP optics; $100 \mathrm{~min}$ total exposure yielded $1.45 \times$ $10^{7}$ photons in the diffraction pattern, with a peak intensity of $6.8 \times 10^{4}$ photons.

At present the detector-sample distance and pixel size determine the upper limit on the crystallite size to approxi-
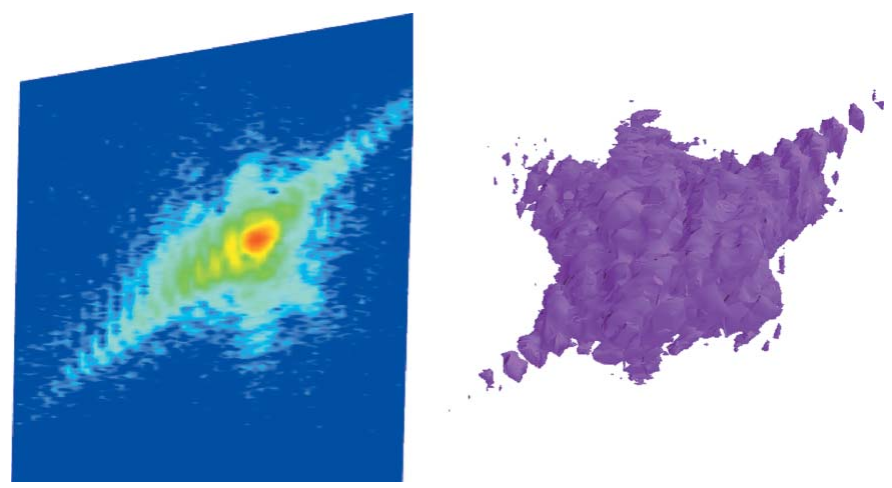

Figure 24

Left: a plane in reciprocal space containing the (111) Bragg peak of a $500 \mathrm{~nm}$ gold crystal. Right: the corresponding isosurface of the local three-dimensional scattering intensity distribution.

mately $500 \mathrm{~nm}$, while the lower limit is set by the flux density; in the unfocused mode, $300 \mathrm{~nm}$ is approximately the lower limit, given the increased exposure time required to count statistically significant scatter. Below this size, focusing optics are mandatory. Implementation of the Eiger detector and an increased detector-arm length will relax the upper limit considerably to closer to two micrometres, at which point the longitudinal coherence length will become the limiting factor.

In the long term, multiple Bragg reflections from the same crystal would provide the full three-dimensional strain tensor (Newton et al., 2010) and could be exploited to characterize the internal structure of devices in situ, during synthesis, and operation in a working environment.

\section{Concluding remarks}

The Materials Science beamline has been upgraded with an undulator X-ray source having the smallest ever reported magnet-pole periodicity of $14 \mathrm{~mm}$ to be routinely used in synchrotron storage rings. This allows photon energies up to nearly $40 \mathrm{keV}$ to be accessible from the medium-energy storage ring of the SLS. New X-ray optics suited to the power density and beam cross-section of the source were designed and installed. The beamline performs close to theoretical values up to approximately $25 \mathrm{keV}$, dropping to approximately $50 \%$ thereafter.

The improved brilliance has resulted in an order-ofmagnitude improvement in diffraction intensity at both the $\mathrm{PD}$ and SD stations. The increased transverse coherence lengths of the undulator radiation has opened up the possibility of performing coherent diffraction imaging in the Bragg geometry.

The authors thank Professor Franz Pfeiffer, Dr Clemens Schulze-Briese and Professor Friso van der Veen for valuable discussions.

\section{References}

Bergamaschi, A., Cervellino, A., Dinapoli, R., Gozzo, F., Henrich, B., Johnson, I., Kraft, P., Mozzanica, A., Schmitt, B. \& Shi, X. (2010). J. Synchrotron Rad. 17, 653-668. 
Bruni, G., Gozzo, F., Capsoni, D., Bini, M., Macchi, P., Simoncic, P., Berbenni, V., Milanese, C., Girella, A., Ferrari, S. \& Marini, A. (2011). J. Pharm. Sci. 100, 2321-2332.

Calvi, M., Schmidt, T., Anghel, A., Cervellino, A., Leake, S. J., Willmott, P. R. \& Tanaka, T. (2013). J. Phys. Conf. Ser. 425, 032017.

Chauvet, C., Polack, F., Silly, M. G., Lagarde, B., Thomasset, M., Kubsky, S., Duval, J. P., Risterucci, P., Pilette, B., Yao, I., Bergeard, N. \& Sirotti, F. (2011). J. Synchrotron Rad. 18, 761-764.

Chubar, O., Elleaume, P. \& Chavanne, J. (1998). J. Synchrotron Rad. 5, 481-484.

Dinapoli, R., Bergamaschi, A., Henrich, B., Horisberger, R., Johnson, I., Kraft, P., Mozzanica, A., Schmitt, B., Shi, X. \& Suter, D. (2010). Nucl. Instrum. Methods Phys. Res. A, 617, 384-386.

Fadenberger, K., Gunduz, I. E., Tsotsos, C., Kokonou, M., Gravini, S., Brandstetter, S., Bergamaschi, A., Schmitt, B., Mayrhofer, P. H., Doumanidis, C. C. \& Rebholz, C. (2010). Appl. Phys. Lett. 97, 144101.

Gozzo, F., Cervellino, A., Leoni, M., Scardi, P., Bergamaschi, A. \& Schmitt, B. (2010). Z. Kristallogr. 225, 616-624.

Gozzo, F., Schmitt, B., Bortolamedi, T., Giannini, C., Guagliadi, A., Lange, M., Meister, D., Maden, D., Willmott, P. \& Patterson, B. D. (2004). J. Alloys Compd. 362, 206-217.

Hara, T., Tanaka, T., Kitamura, H., Bizen, T., Maréchal, X. M., Seike, T., Kohda, T. \& Matsuura, Y. (2004). Phys. Rev. ST Accel. Beams, 7, 050702.

Le Bolloc'h, D., Ravy, S., Dumas, J., Marcus, J., Livet, F., Detlefs, C., Yakhou, F. \& Paolasini, L. (2005). Phys. Rev. Lett. 95, 116401.

Lee, T.-L. \& Zegenhagen, J. (2006). Private communication.

Newton, M. C., Leake, S. J., Harder, R. \& Robinson, I. K. (2010). Nat. Mater. 9, 120-124.

Patterson, B. D., Abela, R., Auderset, H., Chen, Q., Fauth, F., Gozzo, F., Ingold, G., Kühne, H., Lange, M., Maden, D., Meister, D.,
Pattison, P., Schmidt, T., Schmitt, B., Schulze-Briese, C., Shi, M., Stampanoni, M. \& Willmott, P. R. (2005). Nucl. Instrum. Methods Phys. Res. A, 540, 42-67.

Pfeifer, M. A., Williams, G. J., Vartanyants, I. A., Harder, R. \& Robinson, I. K. (2006). Nature (London), 442, 63-66.

Robinson, I. K., Da, Y., Spila, T. \& Greene, J. (2005). J. Phys. D, 38, A7-A10.

Robinson, I. K. \& Harder, R. (2009). Nat. Mater. 8, 291-298.

Rossetti, D., Lienert, U., Pradervand, C., Schneider, R., Shi, M., Zelenika, S., Rossat, M., Hignette, O., Rommeveaux, A. \& SchulzeBriese, C. (2002). Proc. SPIE, 4782, 86-93.

Sayre, D. (1952). Acta Cryst. 5, 843.

Schlepütz, C. M., Herger, R., Willmott, P. R., Patterson, B. D., Bunk, O., Brönnimann, Ch., Henrich, B., Hülsen, G. \& Eikenberry, E. F. (2005). Acta Cryst. A61, 418-425.

Schlepütz, C. M., Mariager, S. O., Pauli, S. A., Feidenhans'l, R. \& Willmott, P. R. (2011). J. Appl. Cryst. 44, 73-83.

Schulte-Schrepping, H., Materlik, G., Heuer, J. \& Teichmann, T. (1995). Rev. Sci. Instrum. 66, 2217-2219.

Schulze, C., Heidenreich, G., Auderset, H., Vermeulen, D. \& Freund, A. K. (1998). Proc. SPIE, 3448, 156-165.

Schulze-Briese, C., Ketterer, B., Pradervand, C., Brönnimann, C., David, C., Horisberger, R., Puig-Molina, A. \& Graafsma, H. (2001). Nucl. Instrum. Methods Phys. Res. A, 467, 230-234.

Slack, G. A. \& Bartram, S. F. (1975). J. Appl. Phys. 46, 89-98.

Stampanoni, M., Groso, A., Isenegger, A., Mikuljan, G., Chen, Q., Meister, D., Lange, M., Betemps, R., Henein, S. \& Abela, R. (2007). AIP Conf. Proc. 879, 848-851.

Willmott, P. R. (2011). An Introduction to Synchrotron Radiation: Techniques and Applications. New York: John Wiley and Sons.

Willmott, P. R., Herger, R., Schlepütz, C. M., Martoccia, D. \& Patterson, B. D. (2005). Synchrotron Radiat. News, 18, 37-42. 